\title{
Adaptive Order WENO Reconstructions for the Semi-Lagrangian Finite Difference Scheme for Advection Problem
}

\author{
Jiajie Chen ${ }^{1}$, Xiaofeng Cai ${ }^{1}$, Jianxian Qiu ${ }^{2}$ and Jing-Mei Qiu ${ }^{1, *}$ \\ ${ }^{1}$ Department of Mathematical Sciences, University of Delaware, Newark, DE, 19717, \\ USA. \\ ${ }^{2}$ School of Mathematical Sciences and Fujian Provincial Key Laboratory of Mathe- \\ matical Modeling and High-Performance Scientific Computing, Xiamen University, \\ Xiamen, Fujian 361005, P.R. China.
}

Received 28 April 2020; Accepted (in revised version) 21 October 2020

\begin{abstract}
We present a new conservative semi-Lagrangian finite difference weighted essentially non-oscillatory scheme with adaptive order. This is an extension of the conservative semi-Lagrangian (SL) finite difference WENO scheme in [Qiu and Shu, JCP, 230 (4) (2011), pp. 863-889], in which linear weights in SL WENO framework were shown to not exist for variable coefficient problems. Hence, the order of accuracy is not optimal from reconstruction stencils. In this paper, we incorporate a recent WENO adaptive order (AO) technique [Balsara et al., JCP, 326 (2016), pp. 780-804] to the SL WENO framework. The new scheme can achieve an optimal high order of accuracy, while maintaining the properties of mass conservation and non-oscillatory capture of solutions from the original SL WENO. The positivity-preserving limiter is further applied to ensure the positivity of solutions. Finally, the scheme is applied to high dimensional problems by a fourth-order dimensional splitting. We demonstrate the effectiveness of the new scheme by extensive numerical tests on linear advection equations, the Vlasov-Poisson system, the guiding center Vlasov model as well as the incompressible Euler equations.
\end{abstract}

AMS subject classifications: 65

Key words: Semi-Lagrangian, weighted essentially nonoscillatory, WENO adaptive order reconstruction, finite difference, mass conservation, Vlasov-Poisson, incompressible Euler.

\section{Introduction}

In this paper, we propose a conservative semi-Lagrangian (SL) finite difference (FD) weighted essentially non-oscillatory adaptive order (WENO-AO) scheme for the advec-

*Corresponding author. Email addresses: jiajie@udel.edu (J. Chen), xfcai@udel.edu (X. Cai), jxqiu@xmu.edu.cn (J. Qiu), jingqiu@udel.edu (J.-M. Qiu) 
tion equation in the form of

$$
u_{t}+\nabla_{\mathbf{x}} \cdot(\mathbf{P}(\mathbf{x}, t) u)=0, \quad(\mathbf{x}, t) \in \mathbb{R}^{d} \times[0, T],
$$

with applications to kinetic equations such as the Vlasov-Poisson (VP) system, the guiding center Vlasov model as well as incompressible Euler equations.

For hyperbolic type advection equations, the Eulerian approach, e.g. Runge-Kutta discontinuous Galerkin (DG) and WENO methods [11], is well-known to be computationally effective with high order accuracy for smooth problems, preservation of local mass conservation, and non-oscillatory capture of shocks. One bottleneck of the Eulerian approach is the stringent Courant-Friedrichs-Lewy (CFL) condition for explicit time stepping. To overcome the CFL condition, the SL approach have been developed. In an SL framework, the solutions is updated by tracking information along characteristics; as a result, the scheme can remain stable for a much larger time stepping size than its Eulerian counterpart. SL algorithms have been applied for a wide range of application fields from climate modeling [27] to kinetic description of plasmas [16,33].

Preservation of mass conservation has been a top priority when designing schemes for hyperbolic conservation laws. For semi-Lagrangian schemes, the conservation property can be assured conveniently in a finite volume $[13,20,23]$ or DG $[8,14,24,30,31]$ frameworks. However, it is nontrivial to design a conservative SL scheme in the FD framework. In [28], a class of high order conservative SL WENO schemes is proposed for advection equations, yet it was shown in the same work there do not exist linear weights in the SL WENO scheme. As a result, the SL WENO scheme cannot achieve the optimal high order accuracy from its reconstruction stencils. To overcome this, we propose to apply other types of WENO methods that use a set of artificial weights and retains high order accuracy. Examples of such WENO reconstructions include CWENO [25, 26], WENOZQ [41], WENO-AO [4], targeted ENO scheme [17,18], hybrid WENO [1,35,40] and their extensions. Especially, there have been systematic studies on WENO-AO [2,3,22]. As in classic WENO-Z [6], smoothness indicators in WENO-ZQ and WENO-AO are properly defined for high order accuracy at critical points. Following the same spirit as the WENO-AO reconstruction [4], we propose new conservative SL FD WENO-AO schemes. The introduction of the WENO-AO procedure enables us to derive explicit formulas of the compact flux reconstruction with the full order in the SL framework. As mentioned in [28], designing the compact flux functions are critical for stability consideration, and designing WENO scheme for the composition of two reconstruction procedures is highly challenging. The proposed schemes in this paper can achieve the formal $(2 r-1)$-th order accuracy when using a stencil of width $(2 r-1)$. For high dimensional problems, we apply a fourth order dimensional splitting $[37,38]$. For problems with positivity physical variable such as the nonlinear VP system, we further apply a positivity preserving (PP) limiter in [36] that maintains high order accuracy of the schemes.

The organization of the paper is as follows: in Section 2, we present the detailed implementation of the SL FD WENO-AO schemes; in Section 3, we demonstrate the effectiveness of the proposed schemes by numerically testing one-dimensional (1D) and 
two-dimensional (2D) linear advection problems, the nonlinear VP system, the guiding center Vlasov model as well as incompressible Euler equations; finally, we conclude in Section 4 .

\section{Semi-Lagrangian finite difference WENO-AO scheme}

We first briefly review the general semi-Lagrangian finite difference framework in [28]. It is denoted as 'SL FD'. Then we will describe the WENO-AO reconstruction procedure [4]. Finally, we use the spirit of WENO-AO reconstruction to propose the new SL FD WENOAO scheme, denoted as 'SL FD WENO-AO'. The complete WENO-AO reconstruction procedure under the SL FD framework will be given in Section 2.3. We will focus on the basic formulation of the 1D advection equation, while a dimensional splitting can be applied to high-dimensional problems.

\subsection{Review of semi-Lagrangian finite difference framework}

We review the SL FD framework for a simple 1D variable coefficient problem

$$
u_{t}+(a(x, t) u)_{x}=0
$$

with the periodic boundary condition. We adopt the following spatial discretization of domain $\left[x_{l}, x_{r}\right]$,

$$
x_{l}=x_{\frac{1}{2}}<x_{\frac{3}{2}}<\cdots<x_{N+\frac{1}{2}}=x_{r},
$$

where the domain is discretized by $N$ uniform grid points with mesh size $\Delta x=\frac{x_{r}-x_{l}}{N}$. We denote $u_{i}^{n}$ as numerical approximation to grid point of $u\left(x_{i}, t^{n}\right)$ at the $n$-th time level. For simplicity, we let $t^{n}=n \Delta t$ with $\Delta t$ being the time stepping size.

The SL FD scheme in [28] is based on integrating the advection equation (2.1) on the time interval $\left[t^{n}, t^{n+1}\right]$,

$$
\begin{aligned}
u\left(x, t^{n+1}\right) & =u\left(x, t^{n}\right)-\left(\int_{t^{n}}^{t^{n+1}} a(x, \tau) u(x, \tau) d \tau\right)_{x} \\
& \doteq u\left(x, t^{n}\right)-\mathcal{F}(x)_{x}
\end{aligned}
$$

where

$$
\mathcal{F}(x) \doteq \int_{t^{n}}^{t^{n+1}} a(x, \tau) u(x, \tau) d \tau .
$$

In order to evaluate $\mathcal{F}(x)_{x}$ by a flux-difference form, as done in a finite difference WENO scheme [21], a sliding average function $\mathcal{H}(x)$ is introduced such that

$$
\mathcal{F}(x)=\frac{1}{\Delta x} \int_{x-\frac{\Delta x}{2}}^{x+\frac{\Delta x}{2}} \mathcal{H}(\xi) d \xi .
$$




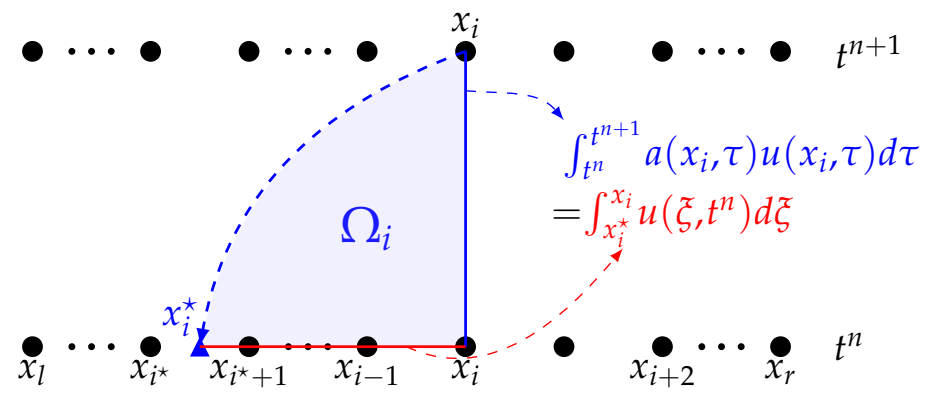

Figure 1: Semi-Lagrangian characteristic tracing.

Taking the derivative of (2.5) gives

$$
\mathcal{F}(x)_{x}=\frac{1}{\Delta x}\left(\mathcal{H}\left(x+\frac{\Delta x}{2}\right)-\mathcal{H}\left(x-\frac{\Delta x}{2}\right)\right) .
$$

In the FD framework, we evaluate Eq. (2.3) at each grid point $x_{i}$,

$$
u_{i}^{n+1}=u_{i}^{n}-\frac{1}{\Delta x}\left(\mathcal{H}\left(x_{i+\frac{1}{2}}\right)-\mathcal{H}\left(x_{i-\frac{1}{2}}\right)\right),
$$

where $\mathcal{H}(x)$ is called the flux function. The flux $\mathcal{H}\left(x_{i+\frac{1}{2}}\right)$ can be reconstructed from its neighboring cell averages of $\mathcal{H}(x)$, i.e.

$$
\overline{\mathcal{H}}_{i} \stackrel{(2.5)}{=} \mathcal{F}\left(x_{i}\right) \stackrel{(2.4)}{=} \int_{t^{n}}^{t^{n+1}} a\left(x_{i}, \tau\right) u\left(x_{i}, \tau\right) d \tau
$$

To evaluate $\overline{\mathcal{H}}_{i}$ from the above equality, we apply the integral form of Eq. (2.1) over the region $\Omega_{i}$ bounded by the three points $\left(x_{i}, t^{n+1}\right),\left(x_{i}, t^{n}\right)$ and $\left(x_{i}^{\star}, t^{n}\right)$. Here $x_{j}^{\star}$ is the foot of characteristics tracing back from the grid point $\left(x_{i}, t^{n+1}\right)$ at the time level $t^{n}$, see Fig. 1 .

In particular, we have

$$
\int_{\Omega_{i}} u_{t}+(a u)_{x} d x d t=0
$$

Applying the divergence Theorem to the above equation, we have

$$
\int_{\Omega_{i}} u_{t}+(a u)_{x}=-\int_{x_{i}^{\star}}^{x_{i}} u\left(\xi, t^{n}\right) d \xi+\int_{t^{n}}^{t^{n+1}} a\left(x_{i}, \tau\right) u\left(x_{i}, \tau\right) d \tau=0 .
$$

Hence,

$$
\overline{\mathcal{H}}_{i}=\int_{t^{n}}^{t^{n+1}} a\left(x_{i}, \tau\right) u\left(x_{i}, \tau\right) d \tau=\int_{x_{i}^{\star}}^{x_{i}} u\left(\xi, t^{n}\right) d \xi,
$$

where $\int_{x_{i}^{\star}}^{x_{i}} u\left(\xi, t^{n}\right) d \xi$ can be reconstructed with high order from grid points values $\left\{u_{i}^{n}\right\}$.

Next, we present the flow chart of the SL FD WENO scheme for updating numerical solutions. The detailed description can be found in [28]. 
Step 1: Locate the feet of characteristics $x_{i}^{\star}$. Track the characteristic backward in time from $\left(x_{i}, t^{n+1}\right)$ to time level $t^{n}$ by solving

$$
\frac{d X(t)}{d t}=a(X(t), t), \quad X\left(t^{n+1}\right)=x_{i}
$$

to locate $X\left(t^{n}\right)=x_{i}^{\star}$. In our numerical experiments, a sixth order Runge-Kutta method is applied to locate $x_{i}^{\star}$.

Step 2: Use the grid point values in the neighborhood of $x_{i}$ to reconstruct the integral $\overline{\mathcal{H}}_{i}=$ $\mathcal{F}\left(x_{i}\right)=\int_{x_{i}^{\star}}^{x_{i}} u\left(\xi, t^{n}\right) d \xi$. We denote this procedure as

$$
\mathcal{F}\left(x_{i}\right) \doteq \mathcal{R}_{1}\left[x_{i}^{\star}, x_{i}\right]\left(u_{i-p_{1}}^{n}, \cdots, u_{i+q_{1}}^{n}\right) .
$$

Step 3: Use $\mathcal{F}\left(x_{i}\right)=\overline{\mathcal{H}}_{i}$ to reconstruct the numerical flux $\mathcal{H}_{i+\frac{1}{2}}$. We denote this reconstruction procedure as

$$
\mathcal{H}_{i+\frac{1}{2}} \doteq \mathcal{R}_{2}\left(\mathcal{F}\left(x_{i-p_{2}}\right), \cdots, \mathcal{F}\left(x_{i+q_{2}}\right)\right) .
$$

The numerical flux $\mathcal{H}_{i+\frac{1}{2}}$ is approximating the flux function $\mathcal{H}\left(x_{i+\frac{1}{2}}\right)$.

Step 4: Update the solution $u_{i}^{n+1}$ by equation (2.6) with the numerical flux reconstructed in Step 3.

As studied in [28], Step 2 and Step 3 can be combined as a single reconstruction procedure $\mathcal{R}=\mathcal{R}_{2} \circ \mathcal{R}_{1}$ with a more compact stencil for numerical stability, i.e.

$$
\mathcal{H}_{i+\frac{1}{2}} \doteq \mathcal{R}\left(u_{i-p}^{n}, \cdots, u_{i+q}^{n}\right) .
$$

In other words, we can reconstruct the numerical flux $\mathcal{H}_{i+\frac{1}{2}}$ from the neighboring point values $\left\{u_{j}^{n}\right\}$ directly.

Remark 2.1. (Nonexistence of linear weights). As found in [28], linear weights for the WENO procedure of $\mathcal{R}$ (2.14) do not exist in general. To show that we use the following example: let $\xi_{j-i}=\frac{x_{j}-x_{j}^{\star}}{\Delta x}, j=i-1, i, i+1$. The reconstruction for $\mathcal{H}\left(x_{i+\frac{1}{2}}\right)$ on two points stencil $\left\{x_{i-1}, x_{i}\right\}$ as in (2.14) is

$$
\mathcal{H}\left(x_{i+\frac{1}{2}}\right)=\Delta x\left(\left(-\frac{1}{2} \xi_{-1}-\frac{1}{4} \xi_{-1}^{2}+\frac{3}{4} \xi_{0}^{2}\right) u_{i-1}^{n}+\left(\frac{1}{4} \xi_{-1}^{2}-\frac{3}{4} \xi_{0}^{2}+\frac{3}{2} \xi_{0}\right) u_{i}^{n}\right) .
$$

The reconstructed flux $\mathcal{H}\left(x_{i+\frac{1}{2}}\right)$ on two points stencil $\left\{x_{i}, x_{i+1}\right\}$ as in (2.14) is given by

$$
\mathcal{H}\left(x_{i+\frac{1}{2}}\right)=\Delta x\left(\left(\frac{1}{2} \xi_{0}+\frac{1}{4} \xi_{0}^{2}+\frac{1}{4} \xi_{1}^{2}\right) u_{i}^{n}+\left(-\frac{1}{4} \xi_{0}^{2}+\frac{1}{2} \xi_{1}-\frac{1}{4} \xi_{1}^{2}\right) u_{i+1}^{n}\right) .
$$


Also, for three points stencil $\left\{x_{i-1}, x_{i}, x_{i+1}\right\}$, we have

$$
\begin{aligned}
\mathcal{H}\left(x_{i+\frac{1}{2}}\right)= & \Delta x\left(\left(-\frac{1}{6} \xi_{-1}-\frac{1}{8} \xi_{-1}^{2}-\frac{1}{36} \xi_{-1}^{3}+\frac{5}{24} \xi_{0}^{2}+\frac{5}{36} \xi_{0}^{3}-\frac{1}{12} \xi_{1}^{2}+\frac{1}{18} \xi_{1}^{3}\right) u_{i-1}^{n}\right. \\
& +\left(\frac{1}{6} \xi_{-1}^{2}+\frac{1}{18} \xi_{-1}^{3}+\frac{5}{6} \xi_{0}-\frac{5}{18} \xi_{0}^{3}+\frac{1}{3} \xi_{1}^{2}-\frac{1}{9} \xi_{1}^{3}\right) u_{i}^{n} \\
& \left.+\left(-\frac{1}{24} \xi_{-1}^{2}-\frac{1}{36} \xi_{-1}^{3}-\frac{5}{24} \xi_{0}^{2}+\frac{5}{36} \xi_{0}^{3}+\frac{1}{3} \xi_{1}-\frac{1}{4} \xi_{1}^{2}+\frac{1}{18} \xi_{1}^{3}\right) u_{i+1}^{n}\right) .
\end{aligned}
$$

In order to apply the classical WENO mechanism, it is desired that there are linear weights $\gamma_{1}$ and $\gamma_{2}$, such that the reconstructed flux function $\mathcal{H}\left(x_{i+\frac{1}{2}}\right)$ in (2.17) is a linear combination of (2.15) and (2.16). In the case of constant coefficient problem $a(x, t) \equiv a$, such linear weights exist; however, for the general variable coefficient situation, $\xi^{\prime}$ s in (2.15)-(2.17) varies and linear weights may fail to exist. For instance, if $\xi_{-1}=\xi_{0}=0$ and $\xi_{1}=1$, then Eqs. (2.15)-(2.17) become

$$
\begin{aligned}
& \mathcal{H}\left(x_{i+\frac{1}{2}}\right)=0 \cdot u_{i-1}^{n}+0 \cdot u_{i}^{n}, \\
& \mathcal{H}\left(x_{i+\frac{1}{2}}\right)=\frac{1}{4} u_{i}^{n}+\frac{1}{4} u_{i+1}^{n}, \\
& \mathcal{H}\left(x_{i+\frac{1}{2}}\right)=-\frac{1}{36} u_{i-1}^{n}+\frac{2}{9} u_{i}^{n}-\frac{1}{36} u_{i+1}^{n} .
\end{aligned}
$$

Linear weights do not exists. In [28], an ENO procedure and a WENO procedure with preset linear weights that do not achieve the optimal accuracy of the full stencil is used.

\subsection{The fifth order WENO-AO reconstruction}

Next, we briefly review the WENO-AO reconstruction procedure, which was first introduced by Balsara, Garaian and Shu [4]. Suppose we have a five point stencil $S^{r 5}=$ $\left\{x_{-2}, x_{-1}, x_{0}, x_{1}, x_{2}\right\}$, with three three-point substencils $S_{1}^{r 3}=\left\{x_{-2}, x_{-1}, x_{0}\right\}, S_{2}^{r 3}=\left\{x_{-1}, x_{0}, x_{1}\right\}$ and $S_{3}^{r 3}=\left\{x_{0}, x_{1}, x_{2}\right\}$. The $i$-th reconstructed polynomial corresponding to stencil $S_{i}^{r 3}$ can be expressed by the Legendre polynomial

$$
P_{i}^{r 3}=u_{0}+u_{a} \mathcal{L}_{1}(x)+u_{b} \mathcal{L}_{2}(x) .
$$

Here Legendre polynomials for the reference domain $\left[-\frac{1}{2}, \frac{1}{2}\right]$, are given by

$$
\begin{aligned}
& \mathcal{L}_{0}(x)=1 ; \\
& \mathcal{L}_{1}(x)=x ; \\
& \mathcal{L}_{2}(x)=x^{2}-\frac{1}{12} ; \\
& \mathcal{L}_{3}(x)=x^{3}-\frac{3}{20} x ; \\
& \mathcal{L}_{4}(x)=x^{4}-\frac{3}{14} x^{2}+\frac{3}{560} .
\end{aligned}
$$


Below are the coefficients $u_{a}$ and $u_{b}$ for stencils $S_{i}^{r 3}, i=1,2,3$, respectively,

$$
\begin{array}{ll}
u_{a}=-2 u_{-1}+u_{-2} / 2+3 u_{0} / 2, & u_{b}=\left(u_{-2}-2 u_{-1}+u_{0}\right) / 2 \\
u_{a}=\left(u_{1}-u_{-1}\right) / 2, & u_{b}=\left(u_{-1}-2 u_{0}+u_{1}\right) / 2 \\
u_{a}=-3 u_{0} / 2+2 u_{1}-u_{2} / 2, & u_{b}=\left(u_{0}-2 u_{1}+u_{2}\right) / 2 .
\end{array}
$$

The smoothness indicator for each of these stencils can be obtained as a scaled sum of the square $L^{2}$ norms of all the derivatives of the relevant reconstructed polynomial [21] and precomputed as

$$
\beta^{r 3}=\left(u_{a}\right)^{2}+\frac{13}{3}\left(u_{b}\right)^{2}
$$

Similarly, the reconstructed polynomial corresponding to stencil $S^{r 5}$ is given by

$$
P^{r 5}=u_{0}+u_{a} \mathcal{L}_{1}(x)+u_{b} \mathcal{L}_{2}(x)+u_{c} \mathcal{L}_{3}(x)+u_{d} \mathcal{L}_{4}(x),
$$

with

$$
\begin{aligned}
& u_{a}=\left(-82 u_{-1}+11 u_{-2}+82 u_{1}-11 u_{2}\right) / 120 \\
& u_{b}=\left(40 u_{-1}-3 u_{-2}-74 u_{0}+40 u_{1}-3 u_{2}\right) / 56, \\
& u_{c}=\left(2 u_{-1}-u_{-2}-2 u_{1}+u_{2}\right) / 12, \\
& u_{d}=\left(-4 u_{-1}+u_{-2}+6 u_{0}-4 u_{1}+u_{2}\right) / 24
\end{aligned}
$$

and the corresponding smoothness indicator

$$
\beta^{r 5}=\left(u_{a}+u_{c} / 10\right)^{2}+\frac{13}{3}\left(u_{b}+\frac{123}{455} u_{d}\right)^{2}+\frac{781}{20}\left(u_{c}\right)^{2}+\frac{1421461}{2275}\left(u_{d}\right)^{2} .
$$

In [4], the linear weights for the stencils $S^{r 5}, S_{1}^{r 3}, S_{2}^{r 3}$, and $S_{3}^{r 3}$ are given by

$$
\begin{array}{ll}
\gamma^{r 5}=\gamma_{H i}, & \gamma_{1}^{r 3}=\left(1-\gamma_{H i}\right)\left(1-\gamma_{L o}\right) / 2, \\
\gamma_{2}^{r 3}=\left(1-\gamma_{H i}\right) \gamma_{L o}, & \gamma_{3}^{r 3}=\left(1-\gamma_{H i}\right)\left(1-\gamma_{L o}\right) / 2 .
\end{array}
$$

Typically, one set $\gamma_{H i} \in[0.85,0.95]$ and $\gamma_{L o} \in[0.85,0.95]$.

Next, we describe the process of obtaining nonlinear weights for fifth order WENO$\mathrm{AO}$ reconstruction. To avoid loss of order at inflection points we use the smoothness indicators to define

$$
\tau=\frac{1}{3}\left(\left|\beta^{r 5}-\beta_{1}^{r 3}\right|+\left|\beta^{r 5}-\beta_{2}^{r 3}\right|+\left|\beta^{r 5}-\beta_{3}^{r 3}\right|\right) .
$$

We obtain the unnormalized nonlinear weights as

$$
\begin{array}{ll}
w^{r 5}=\gamma_{3}^{r 5}\left(1+\tau^{2} /\left(\beta^{r 5}+\epsilon\right)^{2}\right), & w_{1}^{r 3}=\gamma_{1}^{r 3}\left(1+\tau^{2} /\left(\beta_{1}^{r 3}+\epsilon\right)^{2}\right), \\
w_{2}^{r 3}=\gamma_{2}^{r 3}\left(1+\tau^{2} /\left(\beta_{2}^{r 3}+\epsilon\right)^{2}\right), & w_{3}^{r 3}=\gamma_{3}^{r 3}\left(1+\tau^{2} /\left(\beta_{3}^{r 3}+\epsilon\right)^{2}\right),
\end{array}
$$


where $\epsilon$ is a very small number in case the smoothness indicator is zero. Typically $\epsilon \sim$ $10^{-12}$. Then, the normalized weights are given by

$$
\begin{array}{ll}
\bar{w}^{r 5}=w^{r 5} /\left(w^{r 5}+w_{1}^{r 3}+w_{2}^{r 3}+w_{3}^{r 3}\right), & \bar{w}_{1}^{r 3}=w_{1}^{r 3} /\left(w^{r 5}+w_{1}^{r 3}+w_{2}^{r 3}+w_{3}^{r 3}\right), \\
\bar{w}_{2}^{r 3}=w_{2}^{r 3} /\left(w^{r 5}+w_{1}^{r 3}+w_{2}^{r 3}+w_{3}^{r 3}\right), & \bar{w}_{3}^{r 3}=w_{3}^{r 3} /\left(w^{r 5}+w_{1}^{r 3}+w_{2}^{r 3}+w_{3}^{r 3}\right) .
\end{array}
$$

The nonlinear weights and linear weights are combined with reconstructed polynomials as

$$
\begin{aligned}
P(x)= & \frac{\bar{w}^{r 5}}{\gamma^{r 5}}\left(P^{r 5}(x)-\gamma_{1}^{r 3} P_{1}^{r 3}(x)-\gamma_{2}^{r 3} P_{2}^{r 3}(x)-\gamma_{3}^{r 3} P_{3}^{r 3}(x)\right) \\
& +\bar{w}_{1}^{r 3} P_{1}^{r 3}(x)+\bar{w}_{2}^{r 3} P_{2}^{r 3}(x)+\bar{w}_{3}^{r 3} P_{3}^{r 3}(x) .
\end{aligned}
$$

The idea is that when all the smoothness indicators seem to have similar values then only the high order scheme is achieved. Therefore, that when the four smoothness measures associated with these four stencils have similar values, we have $w^{\overline{r 5}} \rightarrow \gamma^{r 5}, w_{1}^{\overline{r 3}} \rightarrow \gamma_{1}^{r 3}$, $w_{2}^{r 3} \rightarrow \gamma_{2}^{r 3}$, and $w_{3}^{r 3} \rightarrow \gamma_{3}^{r 3}$. We then have $P(x) \rightarrow P^{r 5}$.

\subsection{WENO-AO reconstruction procedure for the SL FD scheme}

In this subsection, we will present the detailed WENO-AO reconstruction under the SL FD framework for general variable velocity field $a(x, t)$. Our WENO-AO algorithm also incorporates a flux-based limiter to preserve the positivity of the solution. The algorithm will be extended to a multi-dimensional setting by dimensional splitting.

We first discuss the reconstruction procedure for small time step evolution, i.e. $\mid x_{i}^{\star}-$ $x_{i} \mid \leq \Delta x$ for all $i$. The scheme for extra large time step will be addressed later. Without loss of generality, we assume $a\left(x_{i}, t^{n}\right) \geq 0$ and $x_{i}^{\star} \in\left[x_{i-1}, x_{i}\right]$. The flowchart of the algorithm is summarized as Algorithm 1.

When $a\left(x_{i}, t^{n}\right) \leq 0$, then $x_{i} \leq x_{i}^{\star} \leq x_{i+1}$, a different set of stencils will be chosen with $S_{1}=$ $\left\{x_{i-1}, x_{i}, x_{i+1}, x_{i+2}, x_{i+3}\right\}, S_{2}=\left\{x_{i-1}, x_{i}, x_{i+1}\right\}, S_{3}=\left\{x_{i}, x_{i+1}, x_{i+2}\right\}$, and $S_{4}=\left\{x_{i+1}, x_{i+2}, x_{i+3}\right\}$. We present the reconstructed fluxes $\mathcal{H}^{(k)}\left(x_{i+\frac{1}{2}}\right)$ and smoothness indicators $\beta_{k}$ in Appendix, see Eqs. (A.13)-(A.20).

Here in the first step of Algorithm 1, we derive explicit formulas of $\mathcal{H}^{(k)}\left(x_{i+\frac{1}{2}}\right)$ from $S_{k}=\left\{u_{i-p}, \cdots, u_{i+q}\right\}$ by the following procedure. We first integrate the polynomial interpolating the point values on $S_{k}$ over $\left[x_{j}^{\star}, x_{j}\right]$ (or $\left[x_{j}, x_{j}^{\star}\right]$ depending on the characteristics) as an approximation to $\overline{\mathcal{H}}_{j}$, where $j=i-p, \cdots, i+q$; then $\mathcal{H}^{(k)}\left(x_{i+\frac{1}{2}}\right)$ can be reconstructed from $\left\{\overline{\mathcal{H}}_{j}\right\}_{j=i-p}^{i+q}$ in the classical WENO fashion [21]. Readers can find a detailed description on the reconstruction of $\mathcal{H}^{(k)}\left(x_{i+\frac{1}{2}}\right)$ from [28, section 3.3.1]. Explicit formulas are provided in the Appendix for completeness. Here Eqs. (A.1) and (A.13) were newly derived following the procedure described in [28, section 3.3.1], while Eqs. (A.2)-(A.4) and (A.14)-(A.16) were adopted from [28]. 


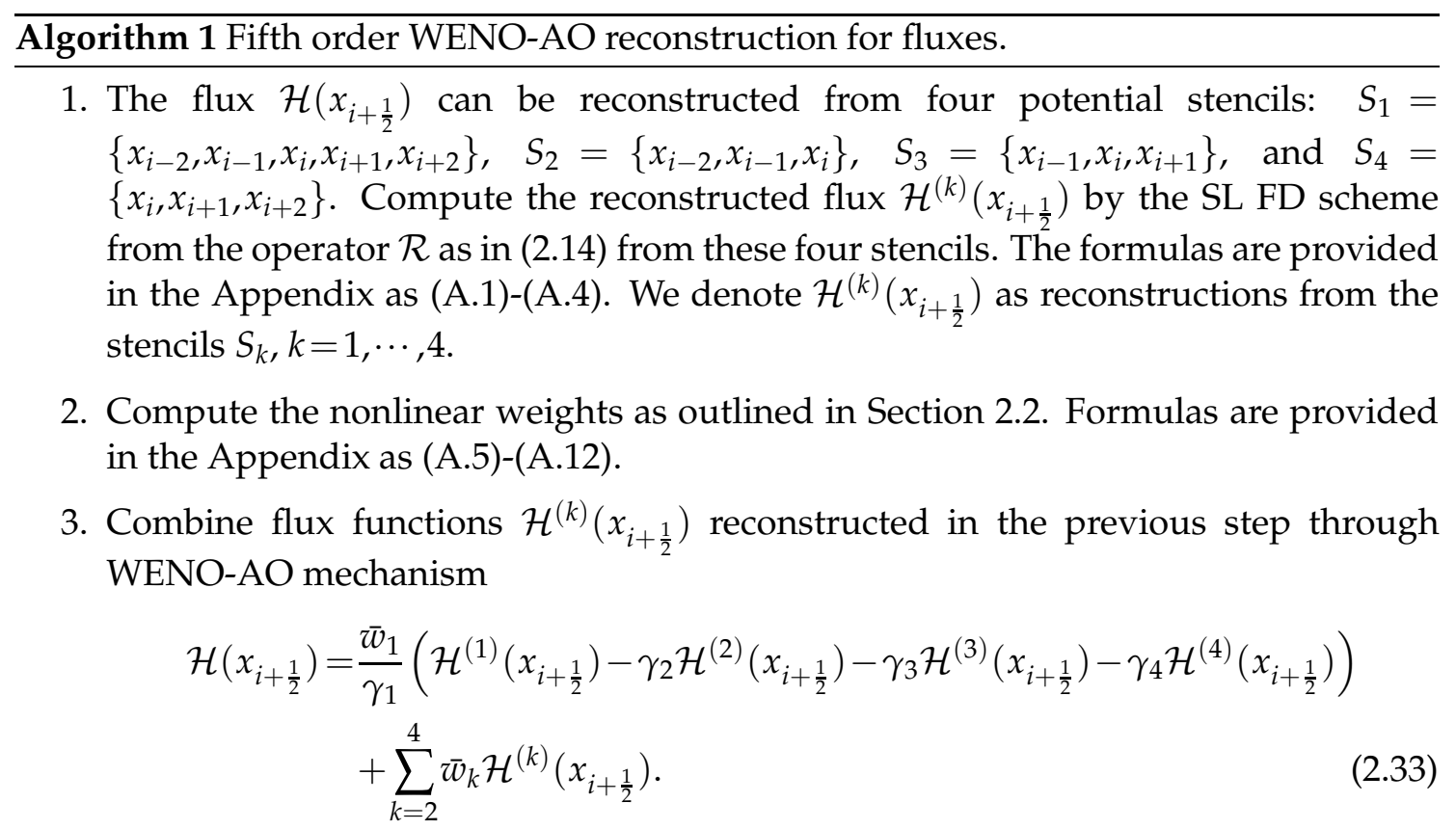

4. The solution is updated by Eq. (2.6).

In general, a large time stepping size is desired for an SL scheme. Again, we assume $a\left(x_{i}, t^{n}\right) \geq 0$ to explain the idea. When the time step is larger than the CFL restriction, we denote $i^{\star}$ as the index such that $x_{i}^{\star} \in\left(x_{i^{\star}-1}, x_{i^{\star}}\right]$. From (2.10) we have

$$
\overline{\mathcal{H}}_{i}=\int_{x_{i}^{\star}}^{x_{i}} u\left(\xi, t^{n}\right) d \xi=\sum_{j=i^{\star}+1}^{i} \int_{x_{j-1}}^{x_{j}} u\left(\xi, t^{n}\right) d \xi+\int_{x_{i}^{\star}}^{x_{i \star}} u\left(\xi, t^{n}\right) d \xi
$$

and flux function

$$
\mathcal{H}\left(x_{i+\frac{1}{2}}\right)=\sum_{j=i^{\star}+1}^{i} \Delta x u_{j}^{n}+\mathcal{R}\left(u_{i^{\star}-p}, \cdots, u_{i^{\star}+q}\right),
$$

where $\mathcal{R}$ is the reconstruction procedure as presented in Algorithm 1. It was pointed out in [28] that when the reconstruction procedure $\mathcal{R}$ is applied to whole grid points, we have the term $\sum_{j=i^{\star}+1}^{i} \Delta x u_{j}^{n}$. Similar procedure applies when $a\left(x_{i}, t^{n}\right) \leq 0$.

To preserve the positivity of the numerical solution, we apply the flux-based limiter for the SL FD scheme as introduced in [36]. The idea of the flux-based limiter is to modify the high order numerical flux $\mathcal{H}_{i+\frac{1}{2}}$ towards the first order monotone flux $h_{i+\frac{1}{2}}$ in a way such that the positivity is preserved. Here the first order monotone flux $h_{i+\frac{1}{2}}$ can be obtained in a similar way as $\mathcal{H}_{i+\frac{1}{2}}$ with first order reconstruction and can be proved to be 
Algorithm 2 SL FD WENO-AO Algorithm with PP limiter under large time stepping size.

Step 1: Locate the foot of each grid point $x_{i}$ along characteristics line from time level $t^{n+1}$ to $t^{n}$. We denote it as $x_{i}^{\star}$. Let $i^{\star}$ to be the index such that $x_{i}^{\star} \in\left(x_{i^{\star}-1}, x_{i^{\star}}\right]$.

Step 2: $\quad$ If $x_{i}^{\star} \leq x_{i}$, let $\xi_{j-i}=\frac{x_{j^{\star}}-x_{j}^{\star}}{\Delta x}, j=i-2, \cdots, i+2$. Compute $\mathcal{H}\left(x_{i+\frac{1}{2}}\right)$ by

$$
\mathcal{H}\left(x_{i+\frac{1}{2}}\right)=\sum_{j=i^{\star}+1}^{i} \Delta x u_{j}^{n}+\mathcal{H}^{\star}\left(x_{i+\frac{1}{2}}\right),
$$

where $\mathcal{H}^{\star}\left(x_{i+\frac{1}{2}}\right)$ is reconstructed from the variables $\left\{u_{i^{\star}-2}, \cdots, u_{i^{\star}+2}\right\}$, as we presented in Algorithm 1.

- If $x_{i}^{\star} \geq x_{i}$, let $\xi_{j-i-1}=\frac{x_{j}^{\star}-x_{j^{\star}-1}}{\Delta x}, j=i-1, \cdots, i+3$. Compute $\mathcal{H}\left(x_{i+\frac{1}{2}}\right)$ by

$$
\mathcal{H}\left(x_{i+\frac{1}{2}}\right)=-\sum_{j=i+1}^{i^{\star}-1} \Delta x u_{j}^{n}+\mathcal{H}^{\star}\left(x_{i+\frac{1}{2}}\right),
$$

where $\mathcal{H}^{\star}\left(x_{i+\frac{1}{2}}\right)$ is reconstructed from the variables $\left\{u_{i^{\star}-1}, \cdots, u_{i^{\star}+3}\right\}$, similar to Algorithm 1 but with different flux functions and smoothness indicators as shown in (A.13)-(A.20).

- Apply the flux-based positivity preserving limiter in the form of (2.37) to modify numerical flux, in order to ensure the positivity preserving property.

Step 3: Update $u_{i}^{n+1}$ by Eq. (2.6) with $\mathcal{H}\left(x_{i \pm \frac{1}{2}}\right)$ computed in Step 2.

positivity preserving. In particular, a modified flux

$$
\tilde{\mathcal{H}}_{i+\frac{1}{2}}=\theta_{i+\frac{1}{2}}\left(\mathcal{H}_{i+\frac{1}{2}}-h_{i+\frac{1}{2}}\right)+h_{i+\frac{1}{2}}
$$

is introduced. Here $\theta_{i+\frac{1}{2}}$ is the parameter to guarantee the positivity preserving property, yet to be as close to the high order numerical flux $\mathcal{H}_{i+\frac{1}{2}}$ as possible. High order accuracy is proved to be maintained in [36]. We omit details of constructing $\theta^{\prime}$ s and in proving the accuracy preserving property for brevity, but refer readers to [36]. We note that we assume that the PDE solution has the positivity preserving property. In fact, for all examples we present in the numerical section, this holds true. The maximum principle preserving property is quite difficult to achieve in a general setting, and we do not consider that in this paper.

An SL FD WENO-AO scheme with positivity preserving property for 1D equation (2.1) follows the flowchart in Algorithm 2. This algorithm allows large time stepping sizes. 


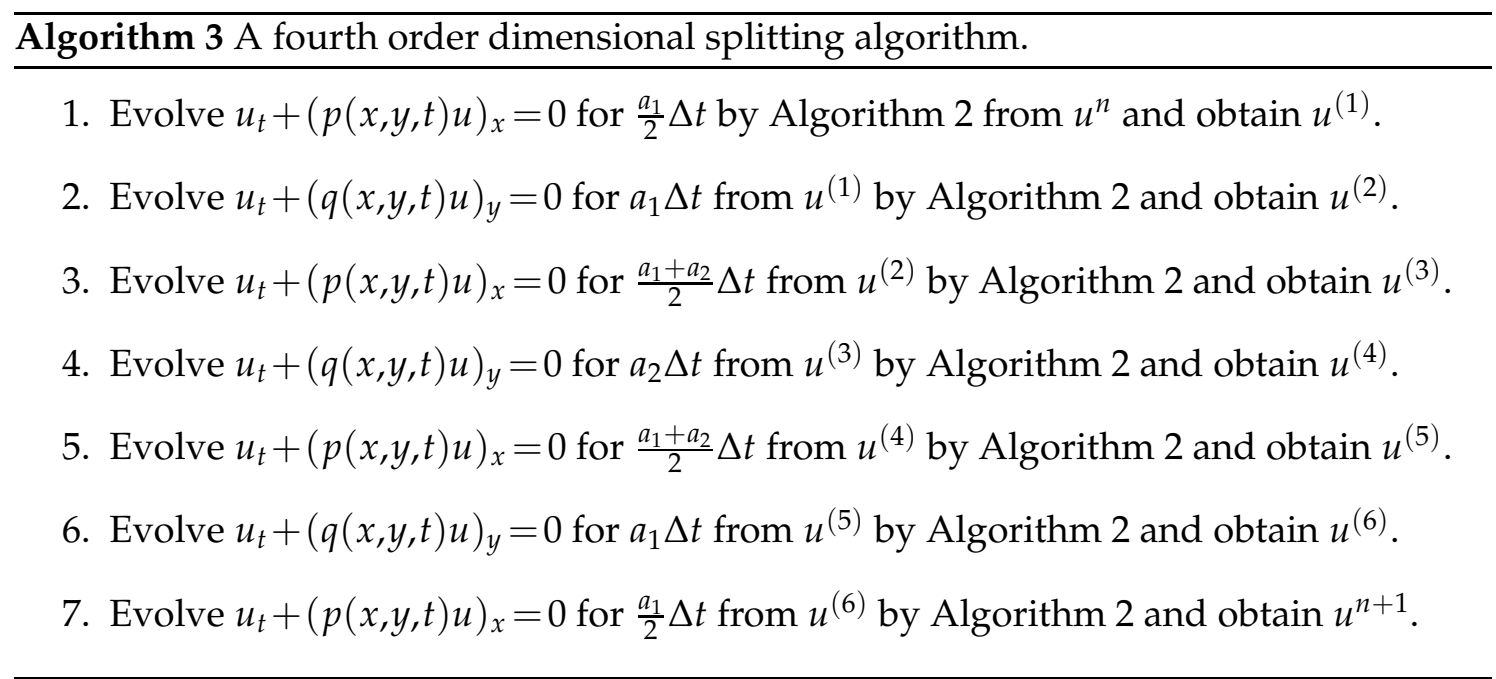

For the 2D problem, we use dimensional-splitting algorithms. A second order Strang splitting [34] and a fourth order splitting [37,38] are used. The flowchart of a fourth order splitting algorithm is summarized in Algorithm 3. We consider a prototype 2D transport equation

$$
u_{t}+(p(x, y, t) u)_{x}+(q(x, y, t) u)_{y}=0 .
$$

For the initialization, we set up parameters $a_{1}=\frac{1}{2-2^{1 / 3}}$ and $a_{2}=-\frac{2^{1 / 3}}{2-2^{1 / 3}}$. Let $\Delta t$ be the standard time stepping size.

\section{Numerical results}

In this section, we present numerical performance of the proposed fifth order SL FD WENO-AO scheme, denoted as WENO-AO. We also present the performance of other semi-Lagrangian WENO schemes for comparison. These schemes are the third order scheme using WENO reconstructions with linear weights $\frac{1}{6}, \frac{2}{3}, \frac{1}{6}$, denoted as WENO3 [28], fifth order WENO reconstructions for constant coefficient problems, denoted as WENO5 [28], and WENO-ZQ scheme [41]. The WENO-ZQ scheme is another reconstruction technique that uses two two-point substencils $S_{l}=\left\{x_{-1}, x_{0}\right\}$ and $S_{r}=\left\{x_{0}, x_{1}\right\}$ and the five-point full stencil. We present its performance for the comparison purpose for some examples. In our numerical tests, we let the time stepping size for 1D and 2D problems as

$$
\Delta t=C F L \cdot \Delta x \quad \text { and } \quad \Delta t=\frac{C F L}{a / \Delta x+b / \Delta y},
$$

respectively. Here $a$ and $b$ are maximum transport speeds in $x$ and $y$ directions, respectively. 


\subsection{D linear transport problems}

Example 3.1. (Linear advection equation). Consider

$$
u_{t}+u_{x}=0, \quad x \in[-1,1] .
$$

We first consider the problem with the initial data $u(x, 0)=\sin (\pi x)$ and its exact solution $u(x, t)=\sin (\pi(x-t))$. We present the $L^{1}$ errors and the spatial orders of convergence of different schemes with a large $C F L=2.2$ in Table 1 . It is observed that all four schemes have reached the expected order of convergence, and that WENO-AO preforms the best in term of the error magnitude especially when the spatial mesh is coarse. Then, we test four schemes with the initial condition which contains a Gaussian, a square wave, a sharp triangle wave, and a half ellipse [21]. Fig. 2 shows the performances of these schemes in capturing solution structures with large time stepping size. We observe that WENO-AO performs the best in capturing discontinuities sharply and without oscillations, while WENO-ZQ produces slightly overshoots around contact discontinuities. The oscillations of WENO-ZQ have been partially discussed in [4, section 5.1].

Table 1: Order of accuracy for (3.1) with $u(x, t=0)=\sin (x)$ at $T=3$. CFL $=2.2$.

\begin{tabular}{||c|cc|cc|cc|cc||}
\hline & \multicolumn{2}{|c|}{ WENO3 } & \multicolumn{2}{c|}{ WENO5 } & \multicolumn{2}{c||}{ WENO-ZQ } & \multicolumn{2}{c||}{ WENO-AO } \\
\hline Mesh & $L^{1}$ error & order & $L^{1}$ error & order & $L^{1}$ error & order & $L^{1}$ error & order \\
\hline 40 & $1.81 \mathrm{e}-05$ & - & $5.96 \mathrm{e}-06$ & - & $1.44 \mathrm{e}-05$ & - & $8.05 \mathrm{e}-07$ & - \\
80 & $1.61 \mathrm{e}-06$ & 3.49 & $1.73 \mathrm{e}-07$ & 5.11 & $2.76 \mathrm{e}-08$ & 9.02 & $2.43 \mathrm{e}-08$ & 5.05 \\
160 & $1.84 \mathrm{e}-07$ & 3.13 & $5.32 \mathrm{e}-09$ & 5.02 & $7.59 \mathrm{e}-10$ & 5.18 & $7.59 \mathrm{e}-10$ & 5.00 \\
320 & $2.24 \mathrm{e}-08$ & 3.04 & $1.65 \mathrm{e}-10$ & 5.01 & $2.37 \mathrm{e}-11$ & 5.11 & $2.37 \mathrm{e}-11$ & 5.00 \\
\hline
\end{tabular}
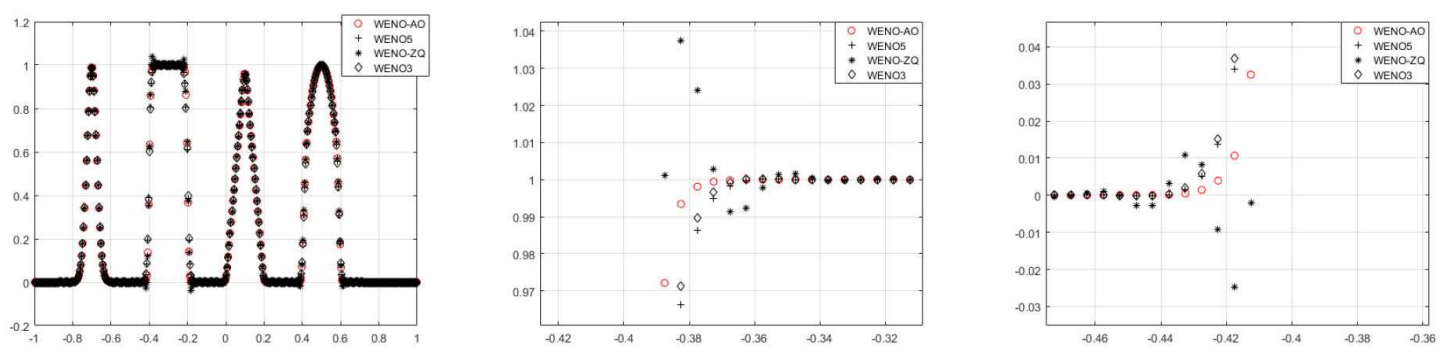

Figure 2: Discontinuous initial value for linear advection problem. $T=4$ and $C F L=2.2$. Middle and right plots are zoom-in plots around the square wave.

Example 3.2. (Linear equation with variable coefficients). Consider

$$
u_{t}+(\sin (x) u)_{x}=0, \quad x \in[0,2 \pi]
$$


Table 2: Order of accuracy for (3.2) with $u(x, t=0)=1$ at $T=1$. CFL $=3$.

\begin{tabular}{||c|cc|cc|cc||}
\hline & \multicolumn{2}{|c|}{ WENO3 } & \multicolumn{2}{c|}{ WENO-ZQ } & \multicolumn{2}{c||}{ WENO-AO } \\
\hline Mesh & $L^{1}$ error & order & $L^{1}$ error & order & $L^{1}$ error & order \\
\hline 40 & $4.61 \mathrm{e}-4$ & - & $1.48 \mathrm{e}-04$ & - & $1.41 \mathrm{e}-04$ & - \\
80 & $2.65 \mathrm{e}-5$ & 4.12 & $7.02 \mathrm{e}-06$ & 4.40 & $6.82 \mathrm{e}-06$ & 4.37 \\
160 & $1.27 \mathrm{e}-6$ & 4.37 & $2.51 \mathrm{e}-07$ & 4.80 & $2.49 \mathrm{e}-07$ & 4.78 \\
320 & $5.89 \mathrm{e}-8$ & 4.44 & $8.31 \mathrm{e}-09$ & 4.93 & $8.29 \mathrm{e}-09$ & 4.91 \\
\hline
\end{tabular}

with the initial data $u(x, t=0)=1$ and the exact solution is $u(x, t)=\frac{\sin \left(2 \arctan \left(e^{-T} \tan (x / 2)\right)\right)}{\sin (x)}$. We apply the SL FD scheme with three different reconstructions: WENO3, WENO-ZQ and WENO-AO for solving this problem up to $T=1$. A large time stepping size with $C F L=3$ is used for all schemes. Table 2 gives $L^{1}$ errors and orders of convergence of these schemes. The expected orders of convergence are achieved. Compared with the WENO3 scheme, WENO-AO and WENO-ZQ both have better performance in error magnitude and order of convergence.

\subsection{D linear transport problems}

From our 1D tests, we observed that WENO-AO has better performance than the WENO$\mathrm{ZQ}$ around contact discontinuities. For the $2 \mathrm{D}$ tests, we only present numerical results of WENO-AO, in comparison with WENO3 and WENO5 from [28]. We use the second order Strang splitting and fourth order dimensional splitting. Unless otherwise specify, we always apply a PP flux limiter.

Example 3.3. (2D linear advection equation). Consider

$$
u_{t}+u_{x}+u_{y}=0, \quad(x, y) \in[0,2 \pi]^{2},
$$

with the initial data $u(x, t=0)=\sin (x+y)$ and with the periodic boundary conditions. We consider the SL FD scheme with three reconstructions, WENO3, WENO5 and WENO$\mathrm{AO}$, and with $C F L=2.2$. Table 3 shows that all three reconstructions have reached the

Table 3: Order of accuracy for (3.3) with $u(x, t=0)=\sin (x+y)$ at $T=3$.

\begin{tabular}{||c|cc|cc|cc||}
\hline & \multicolumn{2}{|c|}{ WENO3 } & \multicolumn{2}{c|}{ WENO5 } & \multicolumn{2}{c||}{ WENO-AO } \\
\hline Mesh & $L^{1}$ error & order & $L^{1}$ error & order & $L^{1}$ error & order \\
\hline $40^{2}$ & $1.14 \mathrm{e}-05$ & - & $4.56 \mathrm{e}-06$ & - & $6.05 \mathrm{e}-07$ & - \\
$80^{2}$ & $7.61 \mathrm{e}-07$ & 3.89 & $1.22 \mathrm{e}-07$ & 5.22 & $1.70 \mathrm{e}-08$ & 5.15 \\
$160^{2}$ & $8.33 \mathrm{e}-08$ & 3.19 & $3.78 \mathrm{e}-09$ & 5.01 & $5.35 \mathrm{e}-10$ & 4.99 \\
$320^{2}$ & $8.97 \mathrm{e}-09$ & 3.22 & $1.12 \mathrm{e}-10$ & 5.07 & $1.60 \mathrm{e}-11$ & 5.06 \\
\hline
\end{tabular}


expected order of convergence. WENO-AO has the best performance in terms of error magnitude.

Example 3.4. (Rigid body rotation problem). Consider

$$
u_{t}-y u_{x}+x u_{y}=0, \quad(x, y) \in[-\pi, \pi]^{2},
$$

with the initial condition,

$$
u(x, y, 0)= \begin{cases}r_{0}^{b} \cos \left(\frac{r^{b}(x) \pi}{2 r_{0}^{b}}\right)^{6}, & \text { if } r^{b}(x)<r_{0}^{b} \\ 0, & \text { otherwise }\end{cases}
$$

where $r_{0}^{b}=0.3 \pi$ and $r^{b}(x)=\sqrt{(x-0.3 \pi)^{2}+y^{2}}$. In Fig. 3, we present the $L^{1}$ error vs. $C F L$ in the logarithm scale. Expected second and fourth order temporal splitting errors are observed, when the second order Strang splitting and the fourth order splitting schemes are applied. It is observed that very large CFL numbers are allowed with numerical stability, and that for large CFL in the range of 10-100, fourth order splitting has better performance in terms of error magnitudes.

We then perform our numerical test using the initial data containing a slotted disk, a cone and a smooth hump, see the top left plot in Fig. 4. A spatial mesh of $60 \times 60$ is used and $C F L=2.2$. Fig. 4 presents the 3D plot of solutions and Fig. 5 presents $1 \mathrm{D}$ cuts of solutions. From these results, we can observe that the solution resolution of WENO$\mathrm{AO}$ seems to be better than that of WENO5; and that WENO-AO with the fourth order splitting performs better than that with Strang splitting when CFL is relatively large. Fig. 6 verifies the schemes' ability in preserving positivity of the solution, by looking into the solutions around zero for schemes with or without PP flux limiter.

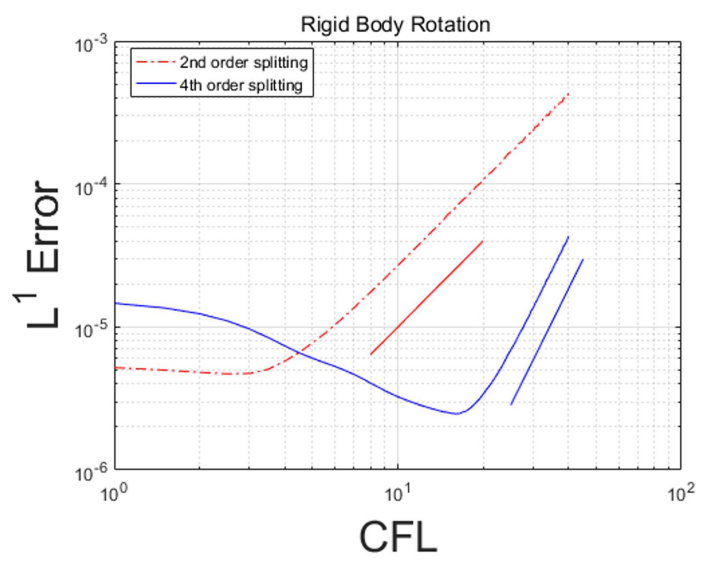

Figure 3: Temporal order of accuracy for (3.4) using WENO-AO with Strang splitting and fourth order splitting. Lines with slope 2 and 4 are plotted as reference. A spatial mesh of $160 \times 160$ is used. 

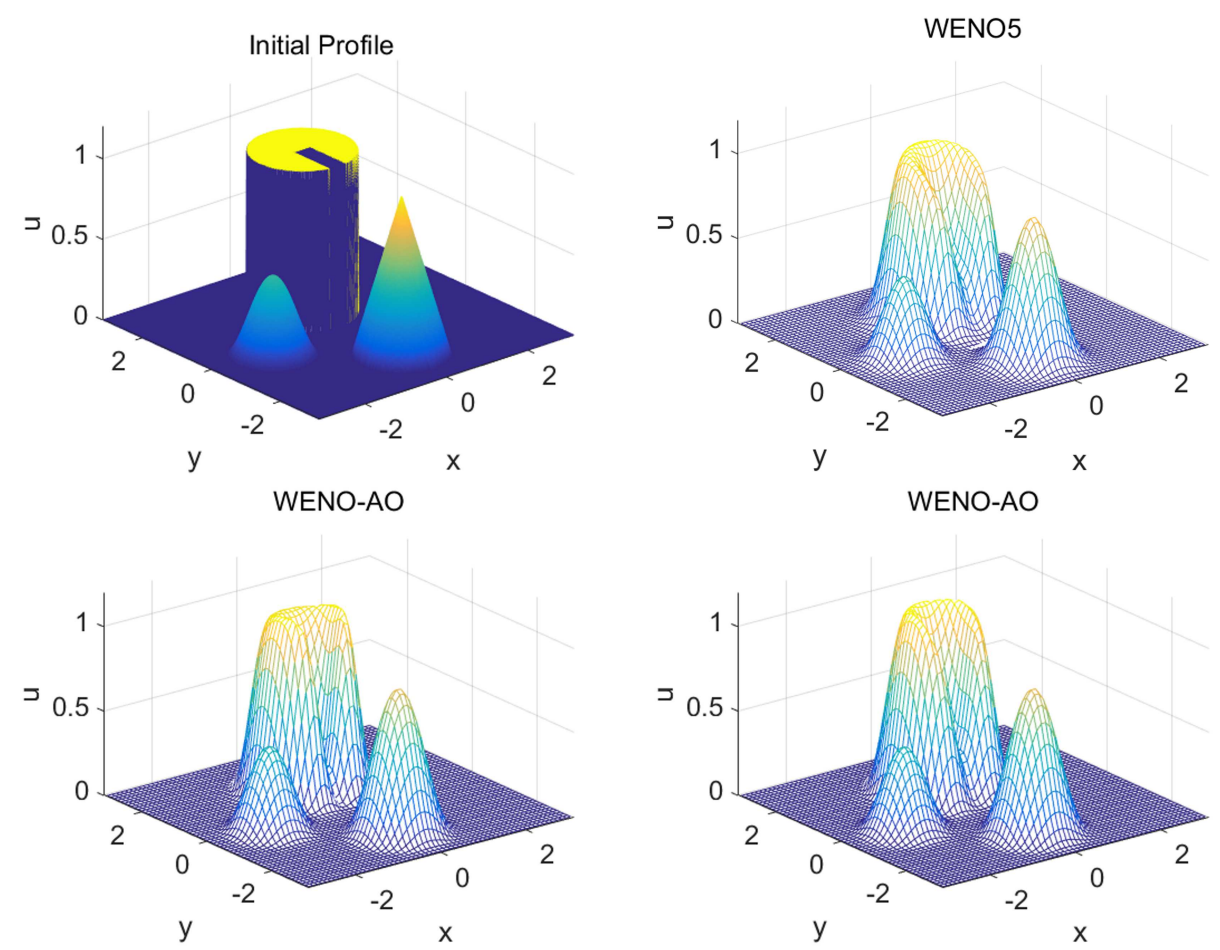

Figure 4: Initial data (upper left), numerical solutions of WENO5 with Strang splitting (upper right), WENO-AO with Strang splitting (lower left) and WENO-AO with fourth order splitting (lower right) for (3.4). $T=12 \pi$.
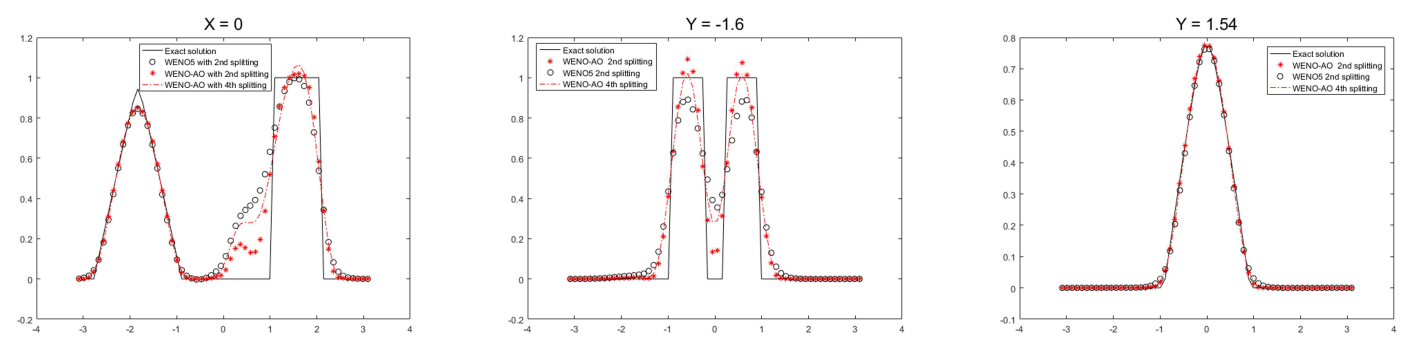

Figure 5: Slides of numerical solution of Fig. 4 at $x=0, y=-1.6$, and $y=1.54$.

Example 3.5. (Swirling deformation problem).

$$
u_{t}-\left(\cos ^{2}\left(\frac{x}{2}\right) \sin (y) g(t) u\right)_{x}+\left(\sin (x) \cos ^{2}\left(\frac{y}{2}\right) g(t) u\right)_{y}=0, \quad(x, y) \in[-\pi, \pi]^{2},
$$

with $g(t)=\cos \left(\frac{\pi t}{T}\right) \pi$. We first test the temporal orders of accuracy of WENO-AO with the Strang splitting and fourth order splitting, using the initial condition (3.5). As shown in Fig. 7, the schemes are stable under very large CFL and expected orders of convergence are observed. 

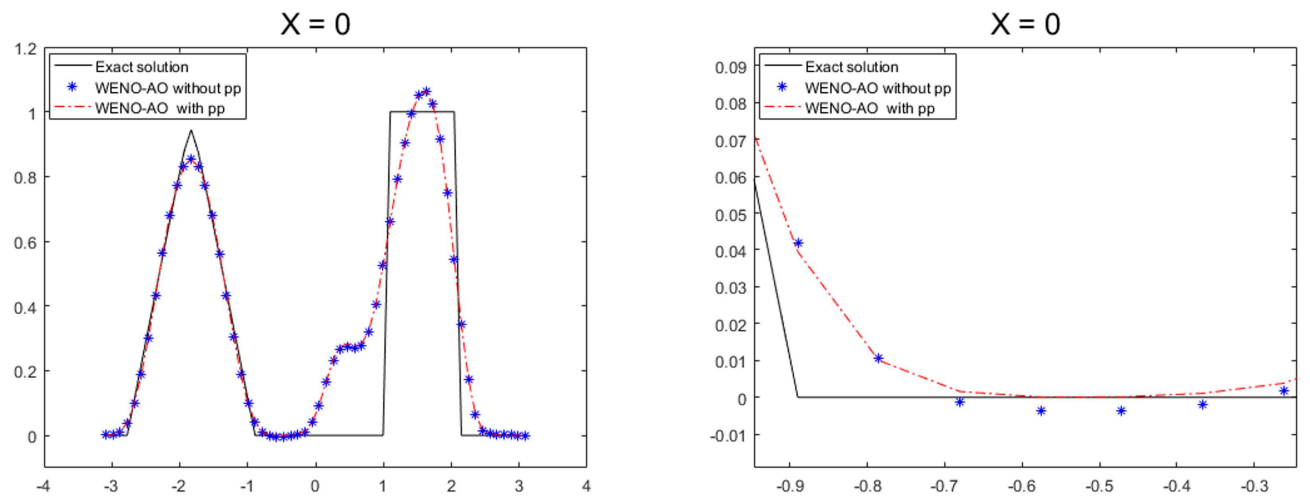

Figure 6: The 1D cuts of the solution of WENO-AO with fourth order splitting with PP limiter and without $\mathrm{PP}$ limiter. $T=12 \pi$.

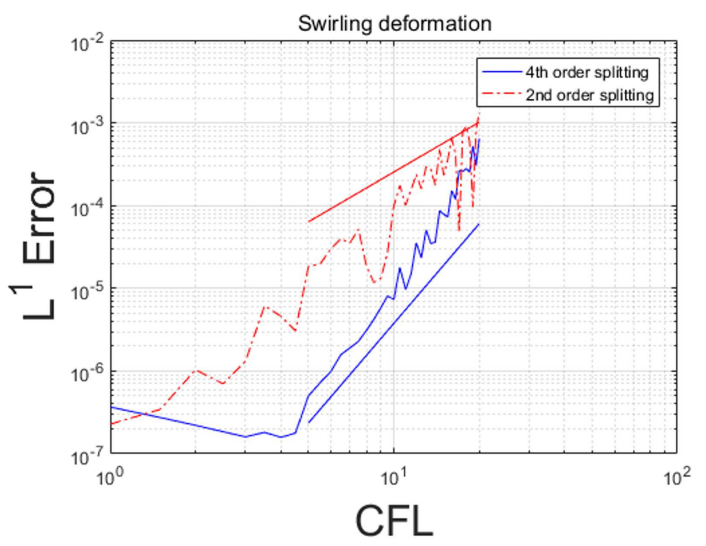

Figure 7: Temporal order of accuracy for (3.6) at $T=1.5$ using Strang splitting and fourth-order splitting. A spatial mesh of $320 \times 320$ is used.

We then test the performance of the scheme using the same initial data as in the previous example. We numerically integrate the solution up to 0.75 , when the initial data are greatly deformed and to time $T=1.5$ when the initial data are recovered. $C F L=2.2$ is used for all simulations. Fig. 8 shows the results of WENO3 with Strang splitting and WENOAO with the fourth order splitting at the final integration time $T=1.5$. 1D cuts of solutions are plotted in Fig. 9 benchmarked with the exact solution. From Figs. 8-9, WENO-AO is observed to perform slightly better than WENO3. Fig. 10 shows the meshes and contour plots of the numerical solution of WENO-AO at final integration time 0.75.

\subsection{Application to nonlinear Vlasov simulations}

Consider the Vlasov-Poisson system 

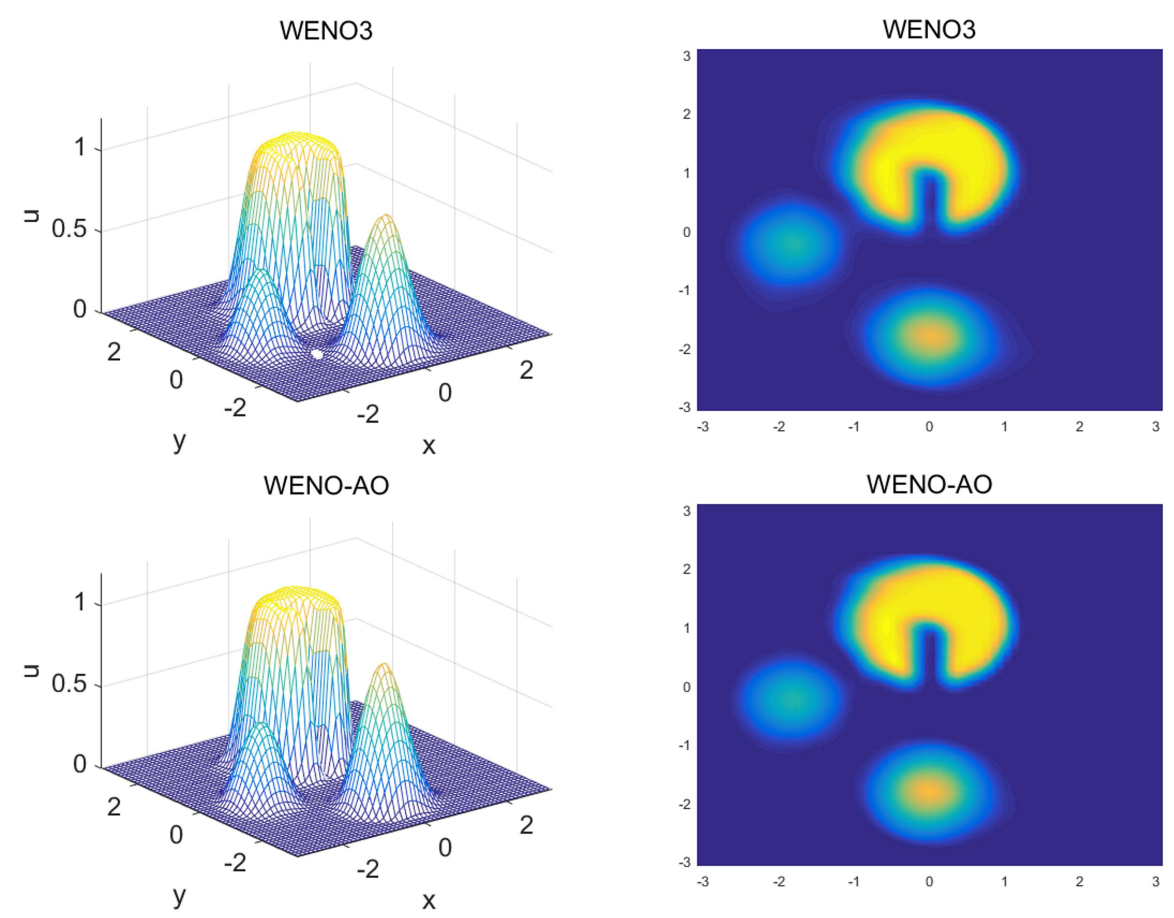

Figure 8: Plots of numerical solution for Eq. (3.6) at $T=1.5$. $C F L=2.2$ and final integration time is 1.5. The mesh of $60 \times 60$ is used.
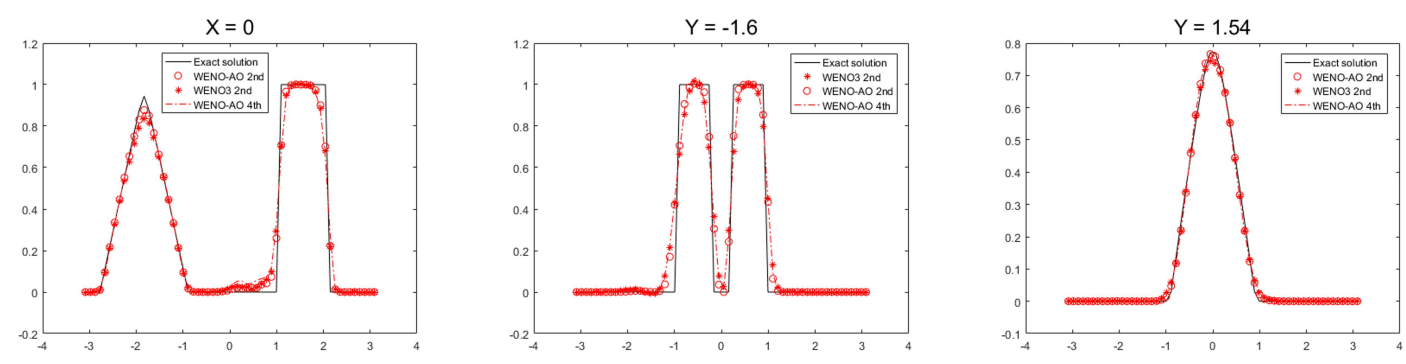

Figure 9: Plots of slides of numerical solution for (3.6) at $x=0, y=-1.6$ and $y=1.54$ with $C F L=2.2$ and final integration time is 1.5 . The mesh of $60 \times 60$ is used.

$$
\begin{aligned}
& \frac{\partial f}{\partial t}+v \cdot \nabla_{x} f+E(t, x) \cdot \nabla_{v} f=0, \quad(x, v) \in \Omega_{x} \times \mathbb{R}, \\
& E(t, x)=-\nabla_{x} \phi(t, x), \quad-\Delta_{x} \phi(t, x)=\rho(t, x) \\
& \rho(t, x)=\int_{\mathbb{R}} f(x, v, t) d v-\frac{1}{\left|\Omega_{x}\right|} \int_{\Omega_{x}} \int_{\mathbb{R}} f\left(t^{0}, x, v\right) d v d x .
\end{aligned}
$$

In (3.7)-(3.9), $x$ is the position, $v$ is velocity, $E$ is the electric field, $\phi$ is the self-consistent electrostatic potential and $f(t, x, v)$ is the probability distribution function. Periodic bound- 

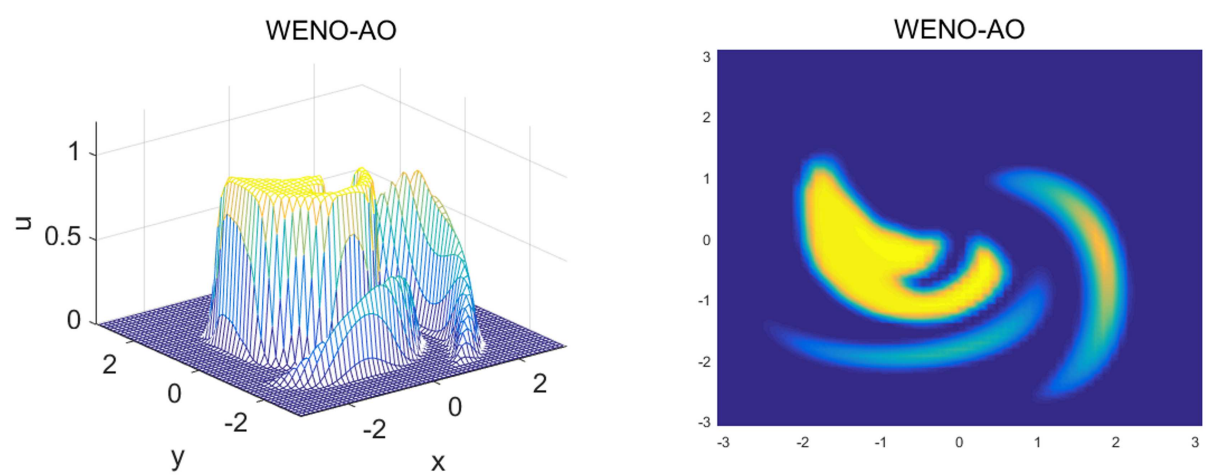

Figure 10: Plots of WENO-AO with fourth order splitting for Eq. (3.6) with $T=1.5 . C F L=1$ and final integration time is 0.75 . The mesh of $60 \times 60$ is used.

ary condition is imposed in the $x$-direction and zero boundary condition is imposed in the $v$-direction for all our tests. A Fast Fourier Transform is used for the Poisson equation. $\rho(t, x)$ is computed by rectangular rule, $\rho(t, x)=\sum_{j} f\left(t, x, v_{j}\right) \Delta v$, which is spectrally accurate [7], when the underlying function is smooth enough. There are some classical preservation results about VP system [29]:

1. the preservation of the $L^{p}$ norm, for $1 \leq p \leq \infty$,

$$
\frac{d}{d t} \int_{v} \int_{x} f(x, v, t)^{p} d x d v=0
$$

2. the preservation of the entropy,

$$
\frac{d}{d t} \int_{v} \int_{x} f(x, v, t) \log (f(x, v, t)) d x d v=0 ;
$$

3. the preservation of the energy,

$$
\frac{d}{d t}\left(\int_{v} \int_{x} f(x, v, t) v^{2} d x d v+\int_{x} E^{2}(x, t) d x\right)=0 .
$$

We would like to emphasize that our new scheme is mass conservative. The other norms preservation is highly nontrivial. Here we track these norms to assess the performance of different numerical schemes. Below we test three different schemes: WENO5 with Strang splitting, WENO-AO with Strang splitting and fourth order splitting to compare their performances. We only apply the positivity-preserving limiter for WENO-AO schemes for all simulations in this subsection.

Example 3.6. (Weak Landau damping). Consider

$$
f(x, v, t=0)=\frac{1}{\sqrt{2 \pi}}(1+\alpha \cos (k x)) \exp \left(\frac{-v^{2}}{2}\right),
$$



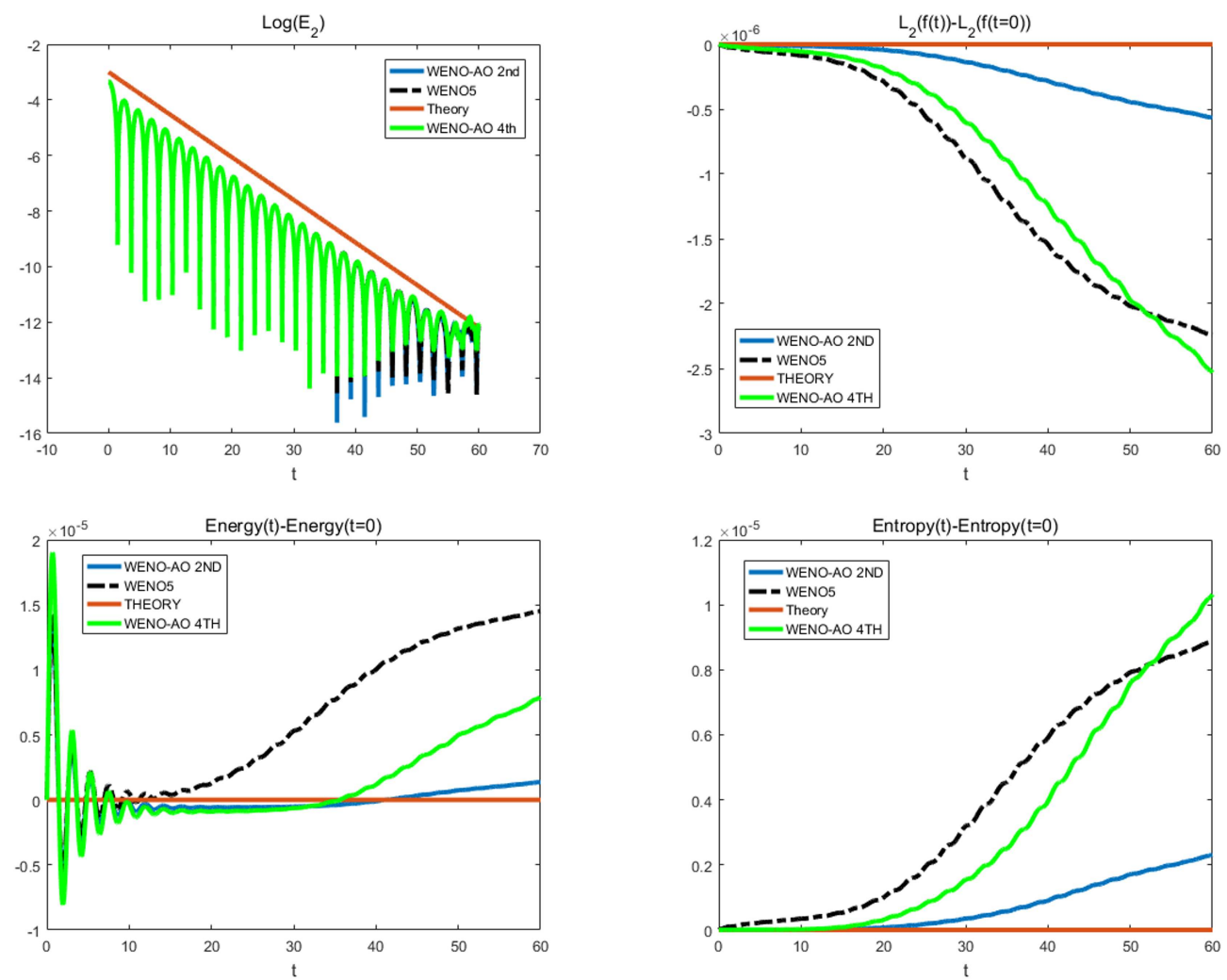

Figure 11: Weak Landau damping: time evolutions of electric field in $L^{2}$ norms, $L^{1}$ norms and $L^{2}$ norms of the solution as well as the discrete kinetic energy and entropy.

with $\alpha=0.01$ and $k=0.5$. The computation domain is $[0,4 \pi] \times\left[-v_{\max }, v_{\max }\right]$. The parameters of numerical simulations are $v_{\max }=5, N_{x}=N_{v}=128, C F L=2.2$ and the final integration time is $T=60$. We observe that all three schemes generate very consistent results. The time evolution of the $L^{2}$ norm of the electric field is plotted in the top left panel of Fig. 11; the correct damping of the electric field is observed, benchmarked with the red line is the theoretical value $\gamma=0.1533$ [15]. The time evolution of variation of the $L^{2}$ norms of solution, energy, and entropy in the discrete sense are also presented. All three schemes have comparable performance on preserving the physical norms. The relevant physical norms are preserved better by WENO-AO than WENO5, especially, WENO-AO with Strang splitting has the best performance on preserving the physical norms. The scheme is mass conservative and positivity preserving, thus preserves the $L^{1}$ norm up to machine precision. We omit this plot for brevity.

Example 3.7. (Strong Landau damping). Consider Eq. (3.13) with the initial condition (3.13) with $\alpha=0.5$ and $k=0.5$. The numerical simulation parameters for all schemes are $v_{\max }=5, N_{x}=N_{v}=128$. We first test the temporal orders of accuracy of WENO-AO with 

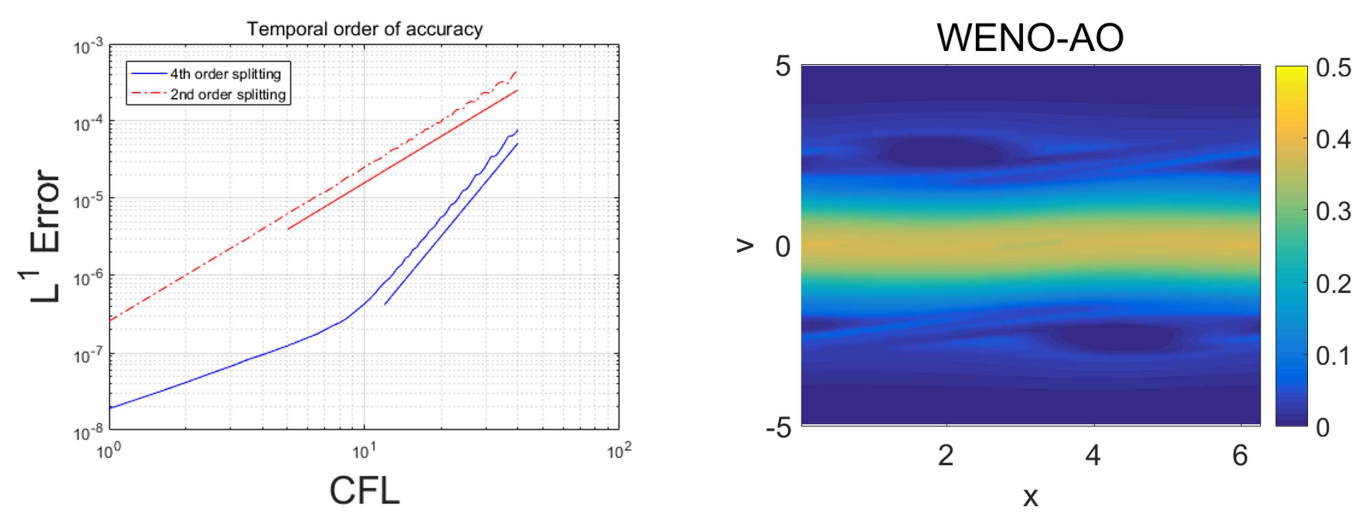

Figure 12: Strong Landau damping. Left: temporal order of accuracy of schemes for (3.13) with the initial condition (3.13) with $\alpha=0.5$ and $k=0.5 . T=2$. The reference solution produced from the same scheme with $C F L=0.01$. Right: surface plots of numerical solutions of WENO-AO with PP limiter at $T=60$ with $C F L=2.2$.

Strang splitting and fourth order splitting; we solve this problem up to $T=2$. From the left plot of Fig. 12, we observe that both schemes have reached expected order. Fourth order splitting scheme has the much smaller error magnitude than the Strang splitting scheme. We then simulate this problem up to $T=60$ and present the surface plot of the solution on the right plot of Fig. 12.

The time evolution of the $L^{2}$ norm of the electric field with the linear decay rate $\gamma_{1}=$ -0.2812 and $\gamma_{2}=0.0770[9,19]$ are plotted in the first plot of Fig. 13. The time evolution of variation of the $L^{2}$ norms of the solution, energy and entropy in the discrete sense are also presented in Fig. 13. All three schemes have comparable performance on preserving these physical invariances. With the mass conservation property and PP flux limiter, the $L^{1}$ norm of the solution is preserved. We skip the plot for brevity.

Example 3.8. (Two stream instability). Consider

$$
f(x, v, t=0)=\frac{2 e^{\frac{-v^{2}}{2}}}{7 \sqrt{2 \pi}}\left(1+5 v^{2}\right)\left(1+\alpha \frac{\cos (2 k x)+\cos (3 k x)}{1.2}+\cos (k x)\right),
$$

with $\alpha=0.01, k=0.5$ on the computation domain $[0,4 \pi] \times\left[-v_{\max }, v_{\max }\right]$. Set our simulation parameters as $v_{\max }=5, N_{x}=N_{v}=128$, and $C F L=2.2$. As in Examples 3.6 and 3.7, we simulate this example up to $T=60$ and report the surface plot of the solution in the first plot of Fig. 14. In Fig. 14, the $L^{2}$ norm of the electric field and the time evolution of $L^{2}$ norms, energy, and entropy of the solutions in the discrete sense are presented. Notice that, the energy and entropy should be preserved theoretically. However, the schemes in this paper only preserve the mass; The deviation of these norms from the exact initial value help to assess the performance of different schemes under the same mesh resolution. 

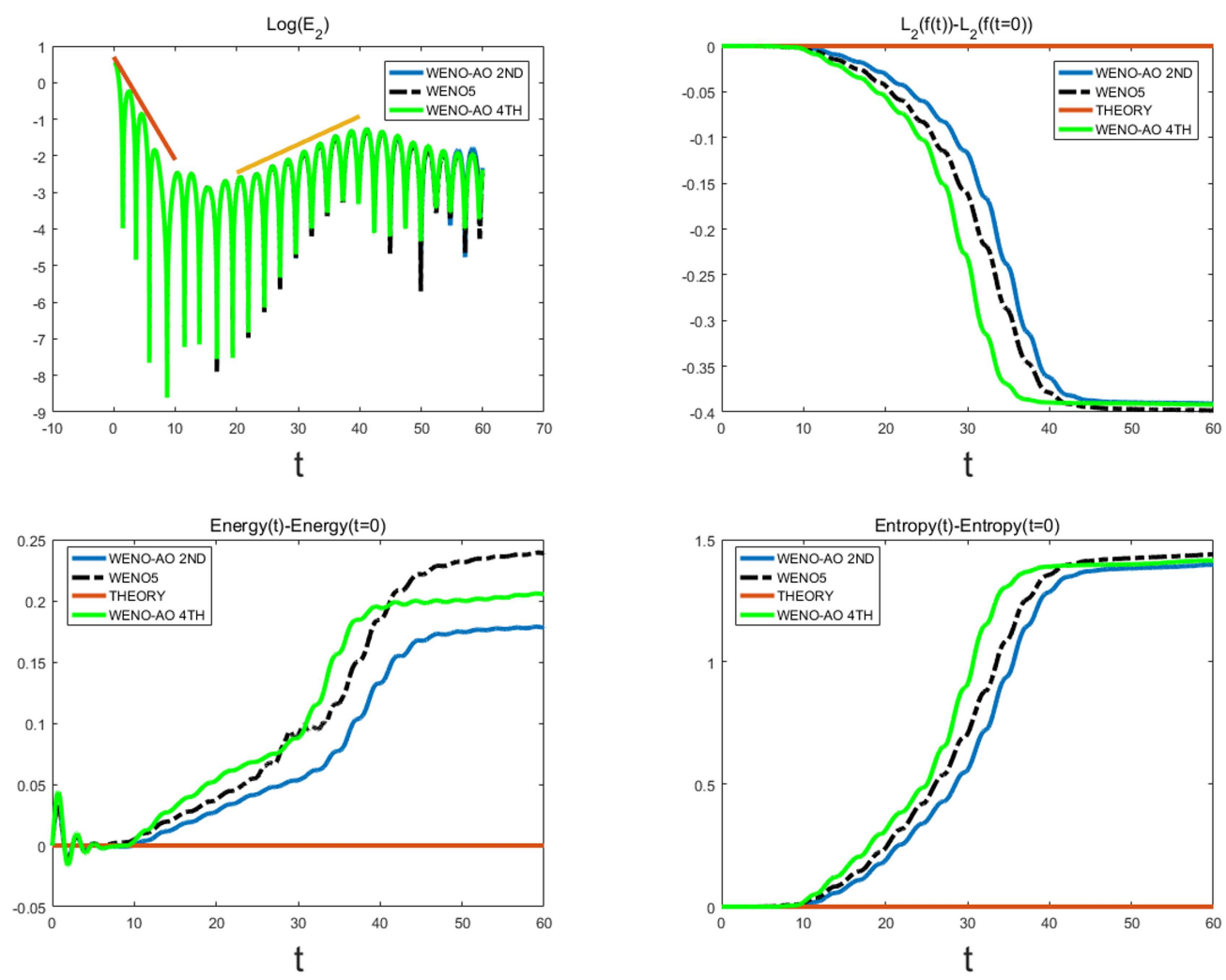

Figure 13: Strong Landau damping: time evolutions of electric field in $L^{2}$ norms, $L^{\infty}$ norms, $L^{1}$ norms and $L^{2}$ norms of the solution as well as the discrete kinetic energy and entropy.

\subsection{Application to 2D guiding center Vlasov model}

Now we consider the guiding center model $[10,12,32]$,

$$
\rho_{t}+E^{\perp} \cdot \nabla \rho=0,
$$

where the unknown variable $\rho$ denotes the charge density of the plasma, $E^{\perp}=\left(-E_{2}, E_{1}\right)$ with the electrostatic field $E=\left(E_{1}, E_{2}\right)$, satisfying

$$
\Delta \Phi=\rho, \quad E=-\nabla \Phi .
$$

It is well known that the $L^{2}$ norms of enstrophy $\rho$ and energy $E$ are constants in time,

$$
\frac{d}{d t}\|\rho(t)\|_{2}=\frac{d}{d t}\|E(t)\|_{2}=0
$$

For this example, classical linear weights for WENO5 do not exist; we will test the WENO$\mathrm{AO}$ and WENO3 schemes and compare their performances. 

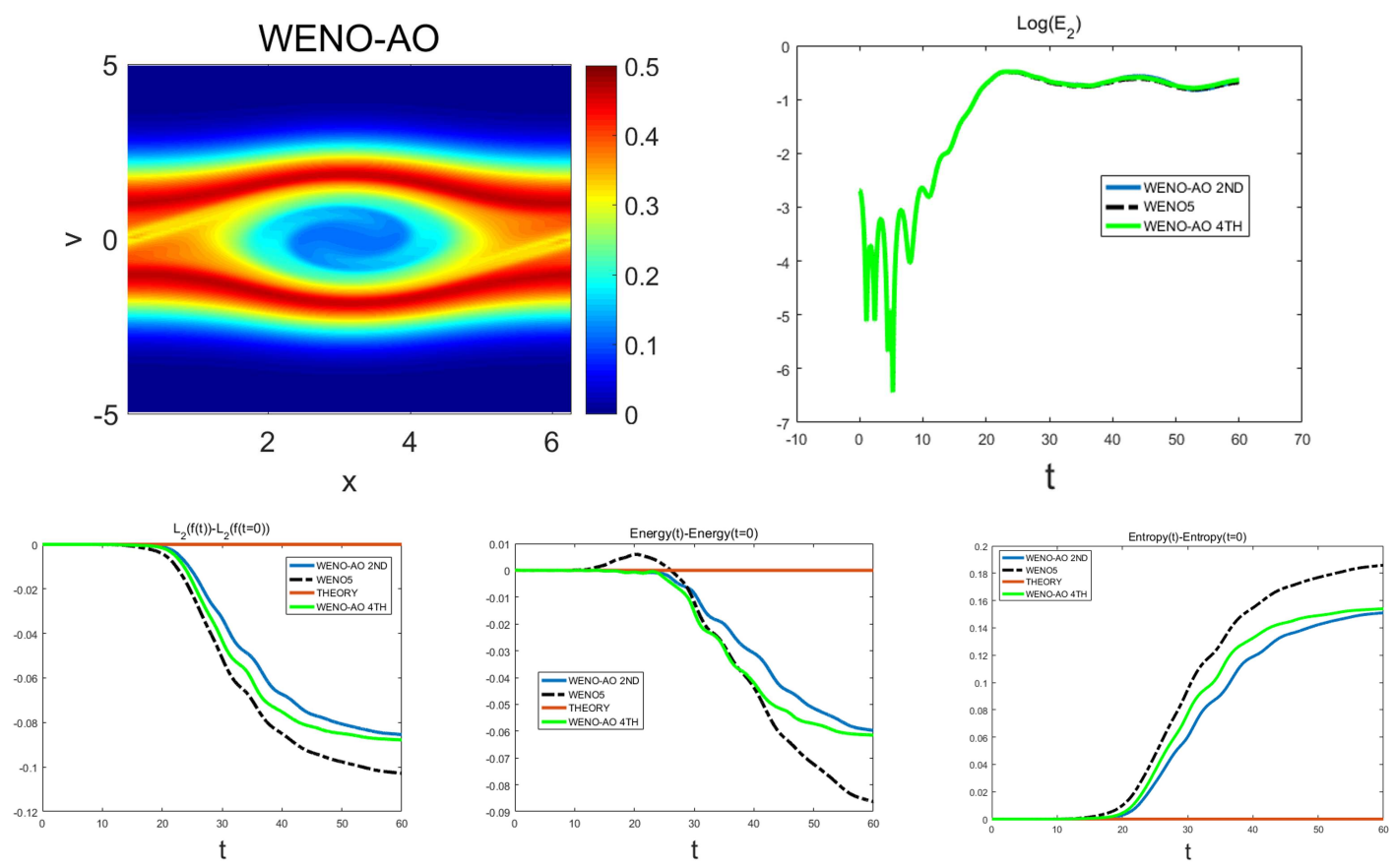

Figure 14: Two stream instability. Upper left: surface plots of the numerical solutions at $T=60$. Time evolutions of electric field in $L^{2}$ norms (upper right), $L^{2}$ norm of the solution, discrete kinetic energy and entropy (bottom plots).

Example 3.9. (Kelvin-Helmholtz instability [10]). We consider (3.15) with the initial condition

$$
\rho(x, y, 0)=\sin (y)+0.015 \cos (x), \quad x \in[0,4 \pi], \quad y \in[0,2 \pi] .
$$

We set $N_{x}=N_{y}=128, C F L=2.2$ and final integration time $T=20$. We applied Strang splitting in time to both WENO3 and WENO-AO. The 2D contour plot of WENO-AO scheme is presented in the first plot of Fig. 15. Then we present a 1D cut of the numerical solutions at $y=\pi$, comparing solutions from WENO3 and WENO-AO. In the second row of Fig. 15, we present the time evolution of the energy and enstrophy in the discrete sense. We observed that WENO-AO scheme is slightly better than the WENO3 scheme. The performance is not significantly better as the errors of both schemes are dominated by the temporal splitting error.

\subsection{Application to the 2D incompressible Euler equations}

We now consider the 2D incompressible Euler equations in the vorticity-stream function,

$$
\begin{aligned}
& \omega_{t}+\nabla \cdot(\mathbf{u} \omega)=0, \\
& \mathbf{u}=\nabla^{\perp} \Phi=\left(-\partial_{y} \Phi, \partial_{x} \Phi\right), \quad \Delta \Phi=\omega .
\end{aligned}
$$



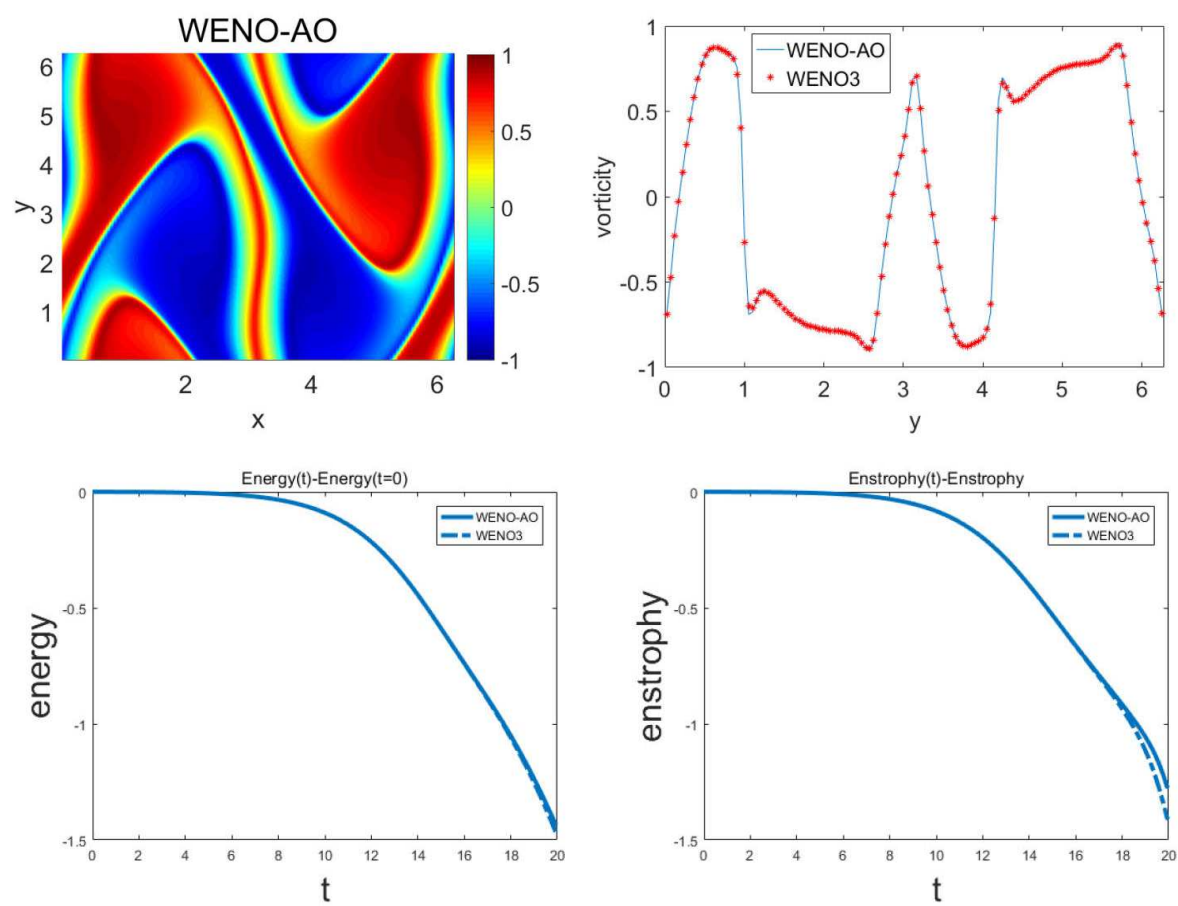

Figure 15: Kelvin-Helmholtz instability: vorticity of WENO-AO at $T=20$ (upper left), $1 \mathrm{D}$ cuts of solutions (upper right), and time evolutions of the energy (lower left) and enstrophy (lower right).

This system preserves the $L^{2}$ norm of enstrophy and $L^{2}$ norm of energy as we described in the guiding center Vlasov model. As we mentioned in Section 3.3, our new scheme is only mass conservative. The other norms preservation is highly nontrivial. Here we track these norms to assess the performance of different numerical schemes. We apply the Strang splitting in time to both WENO3 and WENO-AO.

Example 3.10. (Shear flow problem). We set the initial condition $[5,39]$,

$$
\omega(x, y, 0)= \begin{cases}\delta \cos (x)-\frac{1}{\rho} \operatorname{sech}^{2}((y-\pi / 2) / \rho), & \text { if } y \leq \pi \\ \delta \cos (x)+\frac{1}{\rho} \operatorname{sech}^{2}((3 \pi / 2-y) / \rho), & \text { if } y \geq \pi\end{cases}
$$

with $\delta=0.05$ and $\rho=\pi / 15$. We use $N_{x}=N_{y}=128$ with $C F L=2.2$ and the final integration time $T=8$. The contour plots of both solutions are plotted in the first row of Fig. 16. The variational time evolution of $L^{2}$ norm of enstrophy and energy are plotted in the second row of Fig. 16. We can observe that WENO-AO preserves enstrophy and energy slightly better than WENO3. Both schemes have similar dissipation property since the errors of both schemes are dominated by the splitting errors. 

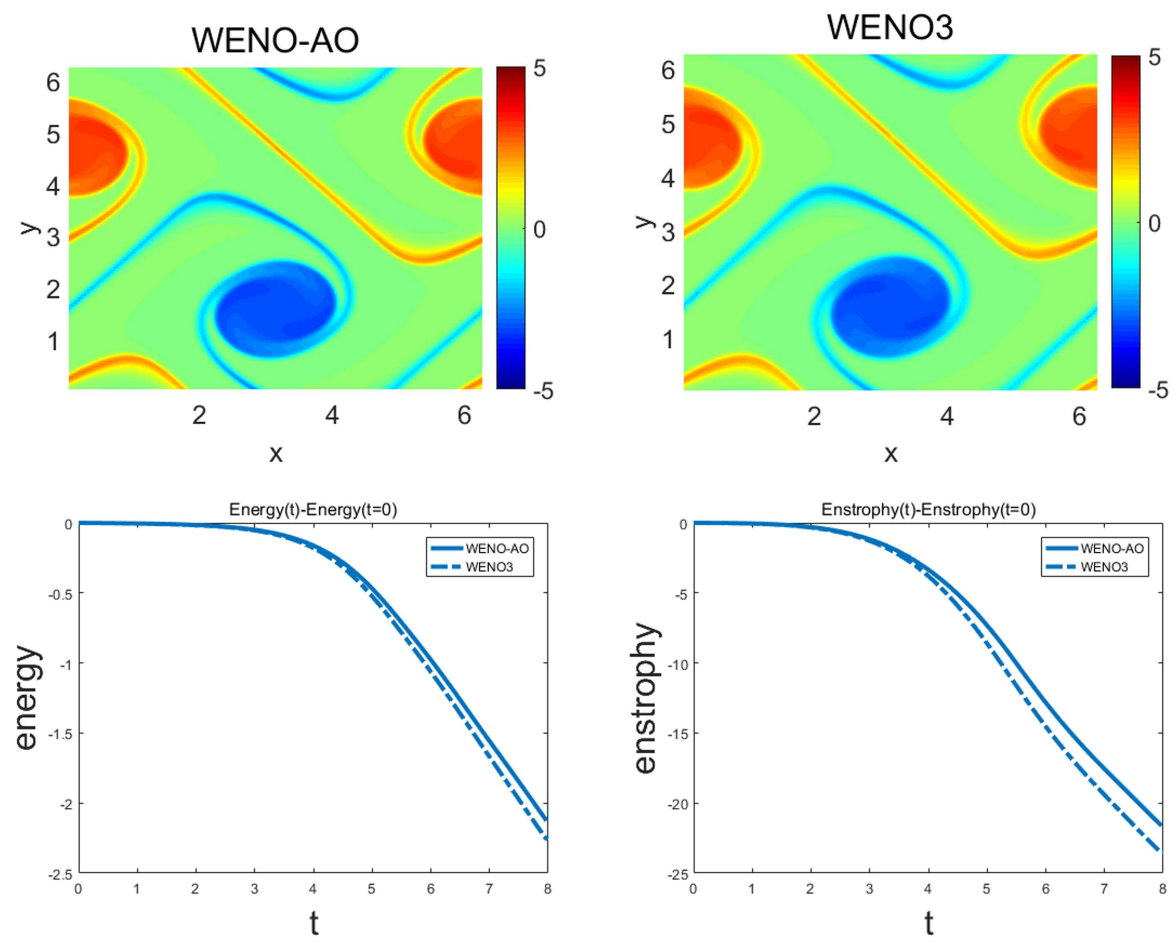

Figure 16: Shear flow problem: vorticity of WENO-AO and WENO3 at $T=8$ with variational time evolutions of the energy and enstrophy.

Example 3.11. (Vortex patch problem). We set the initial condition [28],

$$
\omega(x, y, 0)=\left\{\begin{array}{lll}
-1, & x \in[\pi / 2,3 \pi / 2], & y \in[\pi / 4,3 \pi / 4] \\
1, & x \in[\pi / 2,3 \pi / 2], & y \in[5 \pi / 4,7 \pi / 4]
\end{array}\right.
$$

We set $N_{x}=N_{y}=128, C F L=2.2$ and final integration time $T=10$. The contour WENO-AO solutions and the time evolution of $L^{2}$ norms of enstrophy and energy are plotted in the Fig. 17. The WENO-AO scheme preserves energy and enstrophy norms better. Again, both schemes have roughly similar dissipation property since the errors of both schemes are dominated by the splitting errors.

\section{Acknowledgments}

The research of J. Chen, X. Cai and J.-M. Qiu is supported by NSF grant NSF-DMS1818924, Air Force Office of Scientific Computing FA9550-18-1-0257 and University of Delaware. The research of J. Qiu is supported by NSAF grant U1630247. 

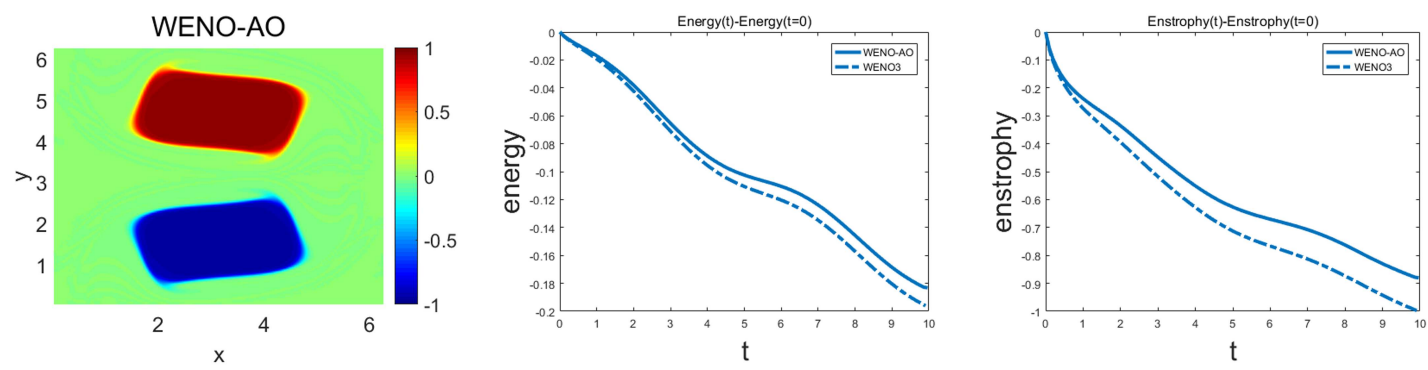

Figure 17: Vortex patch problem. Vorticity of WENO-AO at $T=10$ (left) and time evolution of energy (middle) and enstrophy (right) in the discrete sense.

\section{Appendix}

Flux functions $\mathcal{H}^{(k)}\left(x_{i+\frac{1}{2}}\right)$ for $a\left(x_{i}, t^{n}\right) \geq 0$ : Let $\xi_{j-i}=\frac{x_{j}-x_{j}^{\star}}{\Delta x}, j=i-2, \cdots, i+2$.

$$
\begin{aligned}
& \mathcal{H}^{(1)}\left(x_{i+\frac{1}{2}}\right)=\frac{\Delta x}{43200}\left(\left(1440 \xi_{-2}+1500 \xi_{-2}^{2}+700 \xi_{-2}^{3}+150 \xi_{-2}^{4}+12 \xi_{-2}^{5}-1170 \xi_{-1}^{2}-1430 \xi_{-1}^{3}\right.\right. \\
& -585 \xi_{-1}^{4}-78 \xi_{-1}^{5}-1410 \xi_{0}^{2}-470 \xi_{0}^{3}+705 \xi_{0}^{4}+282 \xi_{0}^{5}+810 \xi_{1}^{2} \\
& \left.-270 \xi_{1}^{3}-405 \xi_{1}^{4}+162 \xi_{1}^{5}+270 \xi_{2}^{2}-330 \xi_{2}^{3}+135 \xi_{2}^{4}-18 \xi_{2}^{5}\right) u_{i-2}^{n} \\
& +\left(-2880 \xi_{-2}^{2}-2080 \xi_{-2}^{3}-540 \xi_{-2}^{4}-48 \xi_{-2}^{5}-9360 \xi_{-1}-3900 \xi_{-1}^{2}+2600 \xi_{-1}^{3}\right. \\
& +1950 \xi_{-1}^{4}+312 \xi_{-1}^{5}+11280 \xi_{0}^{2}+7520 \xi_{0}^{3}-1410 \xi_{0}^{4}-1128 \xi_{0}^{5}-4860 \xi_{1}^{2} \\
& \left.+1080 \xi_{1}^{3}+2430 \xi_{1}^{4}-648 \xi_{1}^{5}-1440 \xi_{2}^{2}+1680 \xi_{2}^{3}-630 \xi_{2}^{4}+72 \xi_{2}^{5}\right) u_{i-1}^{n} \\
& +\left(2160 \xi_{-2}^{2}+2280 \xi_{-2}^{3}+720 \xi_{-2}^{4}+72 \xi_{-2}^{5}+7020 \xi_{-1}^{2}-780 \xi_{-1}^{3}-2340 \xi_{-1}^{4}\right. \\
& -468 \xi_{-1}^{5}+33840 \xi_{0}-14100 \xi_{0}^{3}+1692 \xi_{0}^{5}+14580 \xi_{1}^{2}+1620 \xi_{1}^{3}-4860 \xi_{1}^{4} \\
& \left.+972 \xi_{1}^{5}+3240 \xi_{2}^{2}-3420 \xi_{2}^{3}+1080 \xi_{2}^{4}-108 \xi_{2}^{5}\right) u_{i}^{n} \\
& +\left(-960 \xi_{-2}^{2}-1120 \xi_{-2}^{3}-420 \xi_{-2}^{4}-48 \xi_{-2}^{5}-2340 \xi_{-1}^{2}-520 \xi_{-1}^{3}+1170 \xi_{-1}^{4}\right. \\
& +312 \xi_{-1}^{5}-11280 \xi_{0}^{2}+7520 \xi_{0}^{3}+1410 \xi_{0}^{4}-1128 \xi_{0}^{5}+19440 \xi_{1}-8100 \xi_{1}^{2} \\
& \left.-5400 \xi_{1}^{3}+4050 \xi_{1}^{4}-648 \xi_{1}^{5}-4320 \xi_{-2}^{2}+3120 \xi_{-2}^{3}-810 \xi_{-2}^{4}+72 \xi_{-2}^{5}\right) u_{i+1}^{n} \\
& +\left(180 \xi_{-2}^{2}+220 \xi_{-2}^{3}+90 \xi_{-2}^{4}+12 \xi_{-2}^{5}+390 \xi_{-1}^{2}+130 \xi_{-1}^{3}-195 \xi_{-1}^{4}\right. \\
& -78 \xi_{-1}^{5}+1410 \xi_{0}^{2}-470 \xi_{0}^{3}-705 \xi_{0}^{4}+282 \xi_{0}^{5}-2430 \xi_{1}^{2}+2970 \xi_{1}^{3} \\
& \left.\left.-1215 \xi_{1}^{4}+162 \xi_{1}^{5}-2160 \xi_{2}+2250 \xi_{2}^{2}-1050 \xi_{2}^{3}+225 \xi_{2}^{4}-18 \xi_{2}^{5}\right) u_{i+2}^{n}\right) \text {, } \\
& \mathcal{H}^{(2)}\left(x_{i+\frac{1}{2}}\right)=\Delta x\left(\left(\frac{1}{3} \xi_{-2}+\frac{1}{4} \xi_{-2}^{2}+\frac{1}{18} \xi_{-2}^{3}-\frac{7}{24} \xi_{-1}^{2}-\frac{7}{36} \xi_{-1}^{3}-\frac{11}{24} \xi_{0}^{2}+\frac{11}{36} \xi_{0}^{3}\right) u_{i-2}^{n}\right. \\
& +\left(-\frac{1}{3} \xi_{-2}^{2}-\frac{1}{9} \xi_{-2}^{3}-\frac{7}{6} \xi_{-1}+\frac{7}{18} \xi_{-1}^{3}+\frac{11}{6} \xi_{0}^{2}-\frac{11}{18} \xi_{0}^{3}\right) u_{i}^{n} \\
& \left.+\left(\frac{1}{12} \xi_{-2}^{2}+\frac{1}{18} \xi_{-2}^{3}+\frac{7}{24} \xi_{-1}^{2}-\frac{7}{36} \xi_{-1}^{3}+\frac{11}{6} \xi_{0}-\frac{11}{8} \xi_{0}^{2}+\frac{11}{36} \xi_{0}^{3}\right) u_{i}^{n}\right),
\end{aligned}
$$




$$
\begin{aligned}
\mathcal{H}^{(3)}\left(x_{i+\frac{1}{2}}\right)= & \Delta x\left(\left(-\frac{1}{6} \xi_{-1}-\frac{1}{8} \xi_{-1}^{2}-\frac{1}{36} \xi_{-1}^{3}+\frac{5}{24} \xi_{0}^{2}+\frac{5}{36} \xi_{0}^{3}-\frac{1}{12} \xi_{1}^{2}+\frac{1}{18} \xi_{1}^{3}\right) u_{i-1}^{n}\right. \\
& +\left(\frac{1}{6} \xi_{-1}^{2}+\frac{1}{18} \xi_{-1}^{3}+\frac{5}{6} \xi_{0}-\frac{5}{18} \xi_{0}^{3}+\frac{1}{3} \xi_{1}^{2}-\frac{1}{9} \xi_{1}^{3}\right) u_{i}^{n} \\
& \left.+\left(-\frac{1}{24} \xi_{-1}^{2}-\frac{1}{36} \xi_{-1}^{3}-\frac{5}{24} \xi_{0}^{2}+\frac{5}{36} \xi_{0}^{3}+\frac{1}{3} \xi_{1}-\frac{1}{4} \xi_{1}^{2}+\frac{1}{18} \xi_{1}^{3}\right) u_{i+1}^{n}\right), \\
\mathcal{H}^{(4)}\left(x_{i+\frac{1}{2}}\right)= & \Delta x\left(\left(\frac{1}{3} \xi_{0}+\frac{1}{4} \xi_{0}^{2}+\frac{1}{18} \xi_{0}^{3}+\frac{5}{24} \xi_{1}^{2}+\frac{5}{36} \xi_{1}^{3}+\frac{1}{24} \xi_{2}^{2}-\frac{1}{36} \xi_{2}^{3}\right) u_{i}^{n}\right. \\
& +\left(-\frac{1}{3} \xi_{0}^{2}-\frac{1}{9} \xi_{0}^{3}+\frac{5}{6} \xi_{1}-\frac{5}{18} \xi_{1}^{3}-\frac{1}{6} \xi_{2}^{2}+\frac{1}{18} \xi_{2}^{3}\right) u_{i+1}^{n} \\
& \left.+\left(\frac{1}{12} \xi_{0}^{2}+\frac{1}{18} \xi_{0}^{3}-\frac{5}{24} \xi_{1}^{2}+\frac{5}{36} \xi_{1}^{3}-\frac{1}{6} \xi_{2}+\frac{1}{8} \xi_{2}^{2}-\frac{1}{36} \xi_{2}^{3}\right) u_{i+2}^{n}\right)
\end{aligned}
$$

\section{Nonlinear weights calculation:}

Compute the linear weights as follows: in our implementation, we set $\gamma_{H i}=\gamma_{L o}=0.85$.

$$
\begin{aligned}
& \gamma_{1}=\gamma_{H i}, \quad \gamma_{2}=\left(1-\gamma_{H i}\right)\left(1-\gamma_{L o}\right) / 2, \\
& \gamma_{3}=\left(1-\gamma_{H i}\right) \gamma_{L o}, \quad \gamma_{4}=\left(1-\gamma_{H i}\right)\left(1-\gamma_{L o}\right) / 2 .
\end{aligned}
$$

Compute the smoothness indicator $\beta_{i}$ for each stencil $S_{r}, r=1,2,3,4$ by

$$
\begin{aligned}
\beta_{1}= & \left(\left(-82 u_{i-1}^{n}+11 u_{i-2}^{n}+82 u_{i+1}^{n}-11 u_{i+2}^{n}\right) / 120+\left(2 u_{i-1}^{n}-u_{i-2}^{n}-2 u_{i+1}^{n}+u_{i+2}^{n}\right) / 120\right)^{2} \\
& +\frac{13}{3}\left(\left(40 u_{i-1}^{n}-3 u_{i-2}^{n}-74 u_{i}^{n}+40 u_{i+1}^{n}-3 u_{i+2}^{n}\right) / 56\right. \\
& \left.+\frac{123}{455}\left(-4 u_{i-1}^{n}+u_{i-2}^{n}+6 u_{i}^{n}-4 u_{i+1}^{n}+u_{i+2}^{n}\right) / 24\right)^{2} \\
& +\frac{781}{20}\left(\left(2 u_{i-1}^{n}-u_{i-2}^{n}-2 u_{i+1}^{n}+u_{i+2}^{n}\right) / 12\right)^{2} \\
& +\frac{1421461}{2275}\left(\left(-4 u_{i-1}^{n}+u_{i-2}^{n}+6 u_{i}^{n}-4 u_{i+1}^{n}+u_{i+2}^{n}\right) / 24\right)^{2} \\
\beta_{2}= & \frac{13}{12}\left(u_{i-2}^{n}-2 u_{i-1}^{n}+u_{i}^{n}\right)^{2}+\frac{1}{4}\left(u_{i-2}^{n}-4 u_{i-1}^{n}+3 u_{i}^{n}\right)^{2} \\
\beta_{3}= & \frac{13}{12}\left(u_{i-1}^{n}-2 u_{i}^{n}+u_{i+1}^{n}\right)^{2}+\frac{1}{4}\left(u_{i-1}-u_{i+1}\right)^{2} \\
\beta_{4}= & \frac{13}{12}\left(u_{i}^{n}-2 u_{i+1}^{n}+u_{i+2}^{n}\right)^{2}+\frac{1}{4}\left(3 u_{i}^{n}-4 u_{i+1}^{n}+u_{i+2}^{n}\right)^{2}
\end{aligned}
$$

Compute $\tau$ by

$$
\tau=\frac{1}{3}\left(\left|\beta_{1}-\beta_{2}\right|+\left|\beta_{1}-\beta_{3}\right|+\left|\beta_{1}-\beta_{4}\right|\right) .
$$


Compute the unnormalized nonlinear weights as (A.11), where $\epsilon=10^{-8}$ in our implementation.

$$
w_{i}=\gamma_{i}\left(1+\tau^{2} /\left(\beta_{i}+\epsilon\right)^{2}\right), \quad i=1, \cdots, 4
$$

Normalize the nonlinear weights by

$$
\bar{w}_{i}=w_{i} /\left(w_{1}+w_{2}+w_{3}+w_{4}\right), \quad i=1, \cdots, 4 .
$$

Flux functions $\mathcal{H}^{(k)}\left(x_{i+\frac{1}{2}}\right)$ for $a\left(x_{i}, t^{n}\right) \leq 0$ :

$$
\text { Let } \xi_{j-i-1}=\frac{x_{j}^{\star}-x_{j}}{\Delta x}, j=i-1, \cdots, i+3 \text {. }
$$

$$
\begin{aligned}
& \mathcal{H}^{(1)}\left(x_{i+\frac{1}{2}}\right)=\frac{\Delta x}{43200}\left(\left(-7200 \xi_{-2}+4620 \xi_{-2}^{2}-1420 \xi_{-2}^{3}+210 \xi_{-2}^{4}-12 \xi_{-2}^{5}+9360 \xi_{-1}-9750 \xi_{-1}^{2}\right.\right. \\
& +4550 \xi_{-1}^{3}-975 \xi_{-1}^{4}+78 \xi_{-1}^{5}+4230 \xi_{0}^{2}-5170 \xi_{0}^{3}+2115 \xi_{0}^{4}-282 \xi_{0}^{5} \\
& \left.-810 \xi_{1}^{2}+270 \xi_{1}^{3}+405 \xi_{1}^{4}-162 \xi_{1}^{5}-90 \xi_{2}^{2}-30 \xi_{2}^{3}+45 \xi_{2}^{4}+18 \xi_{2}^{5}\right) u_{i-1}^{n} \\
& +\left(14400 \xi_{-2}-12840 \xi_{-2}^{2}+4720 \xi_{-2}^{3}-780 \xi_{-2}^{4}+48 \xi_{-2}^{5}+18720 \xi_{-1}^{2}-13520 \xi_{-1}^{3}\right. \\
& +3510 \xi_{-1}^{4}-312 \xi_{-1}^{5}-33840 \xi_{0}+14100 \xi_{0}^{2}+9400 \xi_{0}^{3}-7050 \xi_{0}^{4}+1128 \xi_{0}^{5} \\
& \left.+6480 \xi_{1}^{2}-4320 \xi_{1}^{3}-810 \xi_{1}^{4}+648 \xi_{1}^{5}+540 \xi_{2}^{2}+120 \xi_{2}^{3}-270 \xi_{2}^{4}-72 \xi_{2}^{5}\right) u_{i}^{n} \\
& +\left(-14400 \xi_{-2}+14040 \xi_{-2}^{2}-5880 \xi_{-2}^{3}+1080 \xi_{-2}^{4}-72 \xi_{-2}^{5}-14040 \xi_{-1}^{2}+14820 \xi_{-1}^{3}\right. \\
& -4680 \xi_{-1}^{4}+468 \xi_{-1}^{5}-25380 \xi_{0}^{2}-2820 \xi_{0}^{3}+8460 \xi_{0}^{4}-1692 \xi_{0}^{5}-19440 \xi_{1} \\
& \left.+8100 \xi_{1}^{3}-972 \xi_{1}^{5}-1620 \xi_{2}^{2}+180 \xi_{2}^{3}+540 \xi_{2}^{4}+108 \xi_{2}^{5}\right) u_{i+1}^{n} \\
& +\left(7200 \xi_{-2}-7320 \xi_{-2}^{2}+3280 \xi_{-2}^{3}-660 \xi_{-2}^{4}+48 \xi_{-2}^{5}+6240 \xi_{-1}^{2}-7280 \xi_{-1}^{3}\right. \\
& +2730 \xi_{-1}^{4}-312 \xi_{-1}^{5}+8460 \xi_{0}^{2}-1880 \xi_{0}^{3}-4230 \xi_{0}^{4}+1128 \xi_{0}^{5}-6480 \xi_{1}^{2} \\
& \left.-4320 \xi_{1}^{3}+810 \xi_{1}^{4}+648 \xi_{1}^{5}+2160 \xi_{2}+900 \xi_{2}^{2}-600 \xi_{2}^{3}-450 \xi_{2}^{4}-72 \xi_{2}^{5}\right) u_{i+2}^{n} \\
& +\left(-1440 \xi_{-2}+1500 \xi_{-2}^{2}-700 \xi_{-2}^{3}+150 \xi_{-2}^{4}-12 \xi_{-2}^{5}-1170 \xi_{-1}^{2}+1430 \xi_{-1}^{3}\right. \\
& -585 \xi_{-1}^{4}+78 \xi_{-1}^{5}-1410 \xi_{0}^{2}+470 \xi_{0}^{3}+705 \xi_{0}^{4}-282 \xi_{0}^{5}+810 \xi_{1}^{2} \\
& \left.\left.+270 \xi_{1}^{3}-405 \xi_{1}^{4}-162 \xi_{1}^{5}+270 \xi_{2}^{2}+330 \xi_{2}^{3}+135 \xi_{2}^{4}+18 \xi_{2}^{5}\right) u_{i+3}^{n}\right), \\
& \mathcal{H}^{(2)}\left(x_{i+\frac{1}{2}}\right)=\Delta x\left(\left(-\xi_{-2}+\frac{5}{12} \xi_{-2}^{2}-\frac{1}{18} \xi_{-2}^{3}+\frac{7}{6} \xi_{-1}-\frac{7}{8} \xi_{-1}^{2}+\frac{7}{36} \xi_{-1}^{3}+\frac{11}{24} \xi_{0}^{2}-\frac{11}{36} \xi_{0}^{3}\right) u_{i-1}^{n}\right. \\
& +\left(\xi_{-2}-\frac{2}{3} \xi_{-2}^{2}+\frac{1}{9} \xi_{-2}^{3}+\frac{7}{6} \xi_{-1}^{2}-\frac{7}{18} \xi_{-1}^{3}-\frac{11}{6} \xi_{0}+\frac{11}{18} \xi_{0}^{3}\right) u_{i}^{n} \\
& \left.+\left(-\frac{1}{3} \xi_{-2}+\frac{1}{4} \xi_{-2}^{2}-\frac{1}{18} \xi_{-2}^{3}-\frac{7}{24} \xi_{-1}^{2}+\frac{7}{36} \xi_{-1}^{3}-\frac{11}{24} \xi_{0}^{2}-\frac{11}{36} \xi_{0}^{3}\right) u_{i+1}^{n}\right),
\end{aligned}
$$




$$
\begin{aligned}
\mathcal{H}^{(3)}\left(x_{i+\frac{1}{2}}\right)= & -\Delta x\left(\left(-\frac{1}{2} \xi_{-1}+\frac{5}{24} \xi_{-1}^{2}-\frac{1}{36} \xi_{-1}^{3}+\frac{5}{6} \xi_{0}-\frac{5}{8} \xi_{0}^{2}+\frac{5}{36} \xi_{0}^{3}-\frac{1}{12} \xi_{1}^{2}+\frac{1}{18} \xi_{1}^{3}\right) u_{i}^{n}\right. \\
& -\left(\frac{1}{2} \xi_{-1}-\frac{1}{3} \xi_{-1}^{2}+\frac{1}{18} \xi_{-1}^{3}+\frac{5}{6} \xi_{0}^{2}-\frac{5}{18} \xi_{0}^{3}+\frac{1}{3} \xi_{1}-\frac{1}{9} \xi_{1}^{3}\right) u_{i+1}^{n} \\
& \left.-\left(-\frac{1}{6} \xi_{-1}+\frac{1}{8} \xi_{-1}^{2}-\frac{1}{36} \xi_{-1}^{3}-\frac{5}{24} \xi_{0}^{2}+\frac{5}{36} \xi_{0}^{3}+\frac{1}{12} \xi_{1}^{2}+\frac{1}{18} \xi_{1}^{3}\right) u_{i+2}\right), \\
\mathcal{H}^{(4)}\left(x_{i+\frac{1}{2}}\right)= & -\Delta x\left(\left(\xi_{0}-\frac{5}{12} \xi_{0}^{2}+\frac{1}{18} \xi_{0}^{3}+\frac{5}{6} \xi_{1}-\frac{5}{8} \xi_{1}^{2}+\frac{5}{36} \xi_{1}^{3}+\frac{1}{24} \xi_{2}^{2}-\frac{1}{36} \xi_{2}^{3}\right) u_{i+1}^{n}\right. \\
& -\left(-\xi_{0}+\frac{2}{3} \xi_{0}^{2}-\frac{1}{9} \xi_{0}^{3}+\frac{5}{6} \xi_{1}^{2}-\frac{5}{18} \xi_{1}^{3}-\frac{1}{6} \xi_{2}+\frac{1}{18} \xi_{2}^{3}\right) u_{i+2}^{n} \\
& \left.-\left(\frac{1}{3} \xi_{0}-\frac{1}{4} \xi_{0}^{2}+\frac{1}{18} \xi_{0}^{3}-\frac{5}{24} \xi_{1}^{2}+\frac{5}{36} \xi_{1}^{3}-\frac{1}{24} \xi_{2}^{2}-\frac{1}{36} \xi_{2}^{3}\right) u_{i+3}^{n}\right) .
\end{aligned}
$$

Smoothness indicators for $a\left(x_{i}, t^{n}\right) \leq 0$ :

$$
\begin{aligned}
\beta_{1}= & \left(\left(-82 u_{i}^{n}+11 u_{i-1}^{n}+82 u_{i+2}^{n}-11 u_{i+3}^{n}\right) / 120+\left(2 u_{i}^{n}-u_{i-1}^{n}-2 u_{i+2}^{n}+u_{i+3}^{n}\right) / 120\right)^{2} \\
& +\frac{13}{3}\left(\left(40 u_{i}^{n}-3 u_{i-1}^{n}-74 u_{i+1}^{n}+40 u_{i+2}^{n}-3 u_{i+3}^{n}\right) / 56\right. \\
& \left.+\frac{123}{455}\left(-4 u_{i}^{n}+u_{i-1}^{n}+6 u_{i+1}^{n}-4 u_{i+2}^{n}+u_{i+3}^{n}\right) / 24\right)^{2} \\
& +\frac{781}{20}\left(\left(2 u_{i}^{n}-u_{i-1}^{n}-2 u_{i+2}^{n}+u_{i+3}^{n}\right) / 12\right)^{2} \\
& +\frac{1421461}{2275}\left(\left(-4 u_{i}^{n}+u_{i-1}^{n}+6 u_{i+1}^{n}-4 u_{i+2}^{n}+u_{i+3}^{n}\right) / 24\right)^{2} \\
\beta_{2}= & \frac{13}{12}\left(u_{i-1}^{n}-2 u_{i}^{n}+u_{i+1}^{n}\right)^{2}+\frac{1}{4}\left(u_{i-1}^{n}-4 u_{i}^{n}+3 u_{i+1}^{n}\right)^{2} \\
\beta_{3}= & \frac{13}{12}\left(u_{i}^{n}-2 u_{i+1}^{n}+u_{i+2}^{n}\right)^{2}+\frac{1}{4}\left(u_{i}-u_{i+2}\right)^{2} \\
\beta_{4}= & \frac{13}{12}\left(u_{i+1}^{n}-2 u_{i+2}^{n}+u_{i+3}^{n}\right)^{2}+\frac{1}{4}\left(3 u_{i+1}^{n}-4 u_{i+2}^{n}+u_{i+3}^{n}\right)^{2} .
\end{aligned}
$$

\section{References}

[1] A. H. Abdalla, Y. H. Zahran, and A. Kaltayev. A hybrid WENO scheme for conservation laws. Applied Mathematical Sciences, 4(67):3327-3344, 2010.

[2] T. Arbogast, C.-S. Huang, and X. Zhao. Accuracy of WENO and adaptive order WENO reconstructions for solving conservation laws. SIAM Journal on Numerical Analysis, 56(3):1818$1847,2018$.

[3] D. S. Balsara, S. Garain, V. Florinski, and W. Boscheri. An efficient class of WENO schemes with adaptive order for unstructured meshes. Journal of Computational Physics, 404:109062, 2020.

[4] D. S. Balsara, S. Garain, and C.-W. Shu. An efficient class of WENO schemes with adaptive order. Journal of Computational Physics, 326:780-804, 2016. 
[5] J. Bell, P. Colella, and H. Glaz. A second-order projection method for the incompressible Navier-Stokes equations. Journal of Computational Physics, 85(2):257-283, 1989.

[6] R. Borges, M. Carmona, B. Costa, and W. S. Don. An improved weighted essentially non-oscillatory scheme for hyperbolic conservation laws. Journal of Computational Physics, 227(6):3191-3211, 2008.

[7] J. P. Boyd. Chebyshev and Fourier spectral methods. Courier Corporation, 2001.

[8] X. Cai, W. Guo, and J.-M. Qiu. A high order semi-Lagrangian discontinuous Galerkin method for Vlasov-Poisson simulations without operator splitting. Journal of Computational Physics, 354:529-551, 2018.

[9] C.-Z. Cheng and G. Knorr. The integration of the Vlasov equation in configuration space. Journal of Computational Physics, 22(3):330-351, 1976.

[10] A. Christlieb, W. Guo, M. Morton, and J.-M. Qiu. A high order time splitting method based on integral deferred correction for semi-Lagrangian Vlasov simulations. Journal of Computational Physics, 267:7-27, 2014.

[11] B. Cockburn, C. Johnson, C.-W. Shu, and E. Tadmor. Advanced Numerical Approximation of Nonlinear Hyperbolic Equations. Springer, New York, 1998.

[12] N. Crouseilles, M. Mehrenberger, and E. Sonnendrücker. Conservative semi-Lagrangian schemes for Vlasov equations. Journal of Computational Physics, 229(6):1927-1953, 2010.

[13] N. Crouseilles, P. Navaro, and E. Sonnendrücker. Charge-conserving grid based methods for the Vlasov-Maxwell equations. Comptes Rendus Mécanique, 342(10-11):636-646, 2014.

[14] L. Einkemmer. A performance comparison of semi-Lagrangian discontinuous Galerkin and spline based Vlasov solvers in four dimensions. Journal of Computational Physics, 376:937$951,2019$.

[15] F. Filbet and E. Sonnendrucker. Comparison of Eulerian Vlasov solvers. Computer Physics Communications, 150(3):247-266, 2003.

[16] F. Filbet, E. Sonnendrücker, and P. Bertrand. Conservative numerical schemes for the Vlasov equation. Journal of Computational Physics, 172(1):166-187, 2001.

[17] L. Fu, X. Y. Hu, and N. A. Adams. A family of high-order targeted ENO schemes for compressible-fluid simulations. Journal of Computational Physics, 305:333-359, 2016.

[18] L. Fu, X. Y. Hu, and N. A. Adams. A new class of adaptive high-order targeted ENO schemes for hyperbolic conservation laws. Journal of Computational Physics, 374:724-751, 2018.

[19] W. Guo and J.-M. Qiu. Hybrid semi-Lagrangian finite element-finite difference methods for the Vlasov equation. Journal of Computational Physics, 234:108-132, 2013.

[20] C.-S. Huang and T. Arbogast. An Eulerian-Lagrangian Weighted Essentially Nonoscillatory scheme for Nonlinear Conservation Laws. Numerical Methods for Partial Differential Equations, 33(3):651-680, 2017.

[21] G.-S. Jiang and C.-W. Shu. Efficient implementation of weighted ENO schemes. Journal of Computational Physics, 126:202-228, 1996.

[22] R. Kumar and P. Chandrashekar. Simple smoothness indicator and multi-level adaptive order WENO scheme for hyperbolic conservation laws. Journal of Computational Physics, 375:1059-1090, 2018.

[23] P. Lauritzen, R. Nair, and P. Ullrich. A conservative semi-Lagrangian multi-tracer transport scheme (CSLAM) on the cubed-sphere grid. Journal of Computational Physics, 229(5):1401$1424,2010$.

[24] D. Lee, R. Lowrie, M. Petersen, T. Ringler, and M. Hecht. A high order characteristic discontinuous galerkin scheme for advection on unstructured meshes. Journal of Computational Physics, 324:289-302, 2016. 
[25] D. Levy, G. Puppo, and G. Russo. Central WENO schemes for hyperbolic systems of conservation laws. ESAIM: Mathematical Modelling and Numerical Analysis, 33(3):547-571, 1999.

[26] D. Levy, G. Puppo, and G. Russo. Compact central WENO schemes for multidimensional conservation laws. SIAM Journal on Scientific Computing, 22(2):656-672, 2000.

[27] S.-J. Lin and R. B. Rood. Multidimensional flux-form semi-Lagrangian transport schemes. Monthly Weather Review, 124(9):2046-2070, 1996.

[28] J.-M. Qiu and C.-W. Shu. Conservative high order semi-Lagrangian finite difference WENO methods for advection in incompressible flow. Journal of Computational Physics, 230(4):863889, 2011.

[29] J.-M. Qiu and C.-W. Shu. Conservative semi-Lagrangian finite difference WENO formulations with applications to the Vlasov equation. Communications in Computational Physics, 10(4):979, 2011.

[30] J.-M. Qiu and C.-W. Shu. Positivity preserving semi-Lagrangian discontinuous Galerkin formulation: Theoretical analysis and application to the Vlasov-Poisson system. Journal of Computational Physics, 230(23):8386-8409, 2011.

[31] J. A. Rossmanith and D. C. Seal. A positivity-preserving high-order semi-Lagrangian discontinuous Galerkin scheme for the Vlasov-Poisson equations. Journal of Computational Physics, 230(16):6203-6232, 2011.

[32] M. M. Shoucri. A two-level implicit scheme for the numerical solution of the linearized vorticity equation. International Journal for Numerical Methods in Engineering, 17(10):1525$1538,1981$.

[33] E. Sonnendruecker, J. Roche, P. Bertrand, and A. Ghizzo. The semi-Lagrangian method for the numerical resolution of the Vlasov equation. Journal of Computational Physics, 149(2):201220, 1999.

[34] G. Strang. On the construction and comparison of difference schemes. SIAM Journal on Numerical Analysis, 5(3):506-517, 1968.

[35] K. Sung. Troubled-Cell Indicator based on Mean Value Property for Hybrid WENO schemes. Communications in Computational Physics, 27(4):949-975, 2020.

[36] T. Xiong, J.-M. Qiu, Z. Xu, and A. Christlieb. High order maximum principle preserving semi-Lagrangian finite difference WENO schemes for the Vlasov equation. Journal of Computational Physics, 273:618-639, 2014.

[37] H. Yoshida. Construction of higher order symplectic integrators. Physics letters A, 150(57):262-268, 1990.

[38] H. Yoshida. Recent progress in the theory and application of symplectic integrators. In Qualitative and Quantitative Behaviour of Planetary Systems, pages 27-43. Springer, 1993.

[39] X. Zhang and C.-W. Shu. On maximum-principle-satisfying high order schemes for scalar conservation laws. Journal of Computational Physics, 229:3091-3120, 2010.

[40] Z. Zhao, J. Zhu, Y. Chen, and J. Qiu. A new hybrid WENO scheme for hyperbolic conservation laws. Computers \& Fluids, 179:422-436, 2019.

[41] J. Zhu and J. Qiu. A new fifth order finite difference WENO scheme for solving hyperbolic conservation laws. Journal of Computational Physics, 318:110-121, 2016. 